
\title{
3 Research Soure \\ The effect of macrophages polarization on the expression of oxytocin signaling system in enteric neurons
}

\section{Yao Shi}

Shandong University

Shuang Li

Shandong University

Haojie Zhang

Shandong University

Jianchun Zhu

Shandong University

Tongtong Che

University of Jinan

\section{Bing Yan}

Shandong University

Jingxin Li

Shandong University

Chuanyong Liu ( $\sim$ liucy@sdu.edu.cn )

Shandong University School of Medicine: Shandong University Cheeloo College of Medicine

\section{Research}

Keywords: OT signaling system, macrophages polarization, pro-inflammatory and anti-inflammatory factors, neural-immune interactions

Posted Date: June 14th, 2021

DOl: https://doi.org/10.21203/rs.3.rs-611538/v1

License: (c) (i) This work is licensed under a Creative Commons Attribution 4.0 International License. Read Full License 


\section{Abstract}

Background: The aim of the current study is to investigate the effect of macrophages polarization on the expression of oxytocin (OT) and oxytocin receptor (OTR) in enteric neurons.

Methods: In this study, we used a classic colitis model to observe the correlation between macrophages polarization and OT signaling system. In order to further demonstrate the effect of macrophages, we examined the expression of OT signaling system after depletion of macrophages.

Results: The data showed that, in vitro, flowing macrophages polarization to M1 type by LPS, the macrophages supernatant inhibited the expression of OT and OTR in cultured enteric neurons through releasing pro-inflammatory cytokines (IL-1 $\beta$, IL-6 and TNF-a); flowing macrophages polarization to M2 type by IL4, the macrophages supernatant promoted the expression of OT and OTR in cultured enteric neurons through releasing anti-inflammatory cytokine (TGF- $\beta$ ). Furthermore, M1 macrophages decreased the expression of OT signaling system mainly through STAT3/NF-KB pathways in cultured enteric neurons; M2 macrophages increased expression of OT signaling system mainly through activation of Smad2/3 and inhibiting the expression of Peg3 in cultured enteric neurons. In DSS model, we demonstrated that macrophages were polarized to M1 type during the inflammatory phases, the expression of OT and OTR decreased significantly; and macrophages were polarized to M2 type during the recovery phases, the expression of OT and OTR increased significantly. At the same time, we also found that D-mannose increased the expression of OT and OTR through polarization of macrophages to M2 type.

Conclusions: This is the first time to prove that the polarization of macrophages differentially regulates the expression of OT and OTR in enteric neurons.

\section{Introduction}

Oxytocin (OT) is traditionally considered to be a nonapeptide hormone synthesized in the hypothalamus, released from the posterior pituitary into circulation [1, 2], and involved in milk let-down and uterine contraction [3]. In recent years, it is reported that oxytocin receptor (OTR) is widely presents in the central and peripheral systems $[3,4]$. And the role of OT is very broad, it participates in the regulation of certain social behaviors [5], anti-oxidation, proliferation, response, and gastrointestinal immune homeostasis [3]. Studies in our laboratory and other group made it clear that OT is an endogenous neuropeptide in the gastrointestinal tract and regulates the secretion, motility, and immunity of the digestive tract [6-8]. OT mainly expressed in myenteric nerve plexus and submucosal nerve plexus, including neuron bodies and nerve fibers [9].

The intestine is the largest immune organ in the body and contains various innate immune and adaptive immune cells. Macrophages are the most important innate immune cells $[10,11]$ and mainly distributed in the lamina propria and muscular layer. Lamina propria macrophages (LMs) are involved in the regulation of intestinal inflammation and have been identified as the main innate immune cells that 
induced IBD $[12,13]$. Muscular layer macrophages (MMs) are densely distributed and adjacent to enteric neurons and nerve fibers. There is a direct synaptic connection between macrophages and neurons [14]. This connection facilitates the dialogue between ENS and macrophages through neurotransmitters, growth factors, cytokines and hormones [15-17]. Tracey et al. proposed that the excitatory neurotransmitter acetylcholine targeted resident macrophages to inhibit the production of inflammatory mediators [18]. MMs respond to luminal infection by upregulating a neuroprotective program via $\beta 2-$ adrenergic receptor ( $\beta 2-\mathrm{AR})$ signaling and mediate neuronal protection through an arginase 1-(Arg1) polyamine axis [19].

Our previous research also found that OT regulated the polarization of macrophages to relieve intestinal inflammation [20]. OXTR-deficient mice were more susceptible to TNBS- and DSS-associated colitis [21]. It has been reported that local and systemic intestinal OT changes during intestinal inflammation [6, 7]. Therefore, we assumed that the inflammatory factors released by macrophages might regulate the expression of OT and OTR. This article aimed to explore whether the polarization of macrophages regulates the expression of the OT signaling system in ENS which might be of great significance for maintaining intestinal homeostasis.

\section{Materials And Methods}

\subsection{Reagents}

Lipopolysaccharide was purchased from Sigma-Aldrich (StLouis, Ca). Recombinant mouse IL-4, recombinant mouse IL-6, recombinant mouse IL-1 $\beta$, recombinant mouse TNF-a, recombinant mouse IL10 , and recombinant mouse TGF- $\beta$ were purchased from R\&D Systems (Minneapolis, MN). Tocilizumab, LY2109761, R-7050, Stattic, Bay 11-7082, SIS3HCl were purchased from Selleck (Huston, USA). IL1Ra was purchased from Make Research Easy (Nanjing, China). Monoclonal mouse anti- $\beta$-actin (TA-09) from Zhongshan Golden Bridge Biotechnology (Beijing, China). Antibodies for OT, OTR, $\beta$-Tubulin, CD206, F480, phospho-STAT3, STAT3, phosphor-SMAD2, SMAD2, phosphor-SMAD3, SMAD3, p65, and phosphorp65 were from Abcam (Cambridge, UK) and Cell Signaling Technology (Danvers, MA). The secondary antibodies were purchased from Invitrogen Life Technology (Foster City, CA). Mouse ELISA kits were obtained from R\&D Systems and CUSABIO (Wuhan, China). All reagents were analytical grade.

\subsection{Cell isolation and culture}

RAW264.7 macrophage-like cell line were purchased from the Cell Bank of Chinese Academy of Science (Shanghai, China).

The enteric neurons were obtained from C57BL6/J mice as previously described [22]. Briefly, the mice were euthanized with no pain and cut the outer skin of the peritoneum. The colon tissue was cut out 3-5 $\mathrm{cm}$ under a microscope. The colon tissue was immediately placed into a silgard lined dissecting dish containing carbogen gassed Krebs saline of the following composition (in $\mathrm{mM}$ ): $\mathrm{NaCl} 120.6, \mathrm{KCl} 5.9$, $\mathrm{CaCl}_{2} 2.5, \mathrm{KH}_{2} \mathrm{PO}_{4} 1.2, \mathrm{MgCl}_{2} 1.2, \mathrm{NaHCO}_{3}$ 15.4. The mucosa, submucosa, circular muscle and serosa 
layers were removed by dissection, leaving the myenteric plexus exposed. Then this longitudinal muscle myenteric plexus (LMMP) preparations were incubated in Krebs saline containing papain $(10 \mathrm{mg} / \mathrm{ml})$ (P8150, Solarbio, Beijing, China) at $37^{\circ} \mathrm{C}$ for $50 \mathrm{~min}$. The tissues were stretched as much as possible under the microscope. The LMMP were washed three times with $4 \mathrm{ml}$ ice-cold PBS and Dulbecco's modified Eagle's medium. Immediately, the tissue was cut with scissors and digested with $1 \mathrm{mg} / \mathrm{ml}$ Collagenase II (17101-017, Invitrogen) in a humidified incubator (Thermo Forma, Hamilton, USA) with 5\% $\mathrm{CO}_{2}$ at $37^{\circ} \mathrm{C}$ for $55 \mathrm{~min}$, after centrifugation, the enteric neurons were cultured in Dulbecco's modified Eagl e's medium (Gibco, Foster City, CA) supplemented 10\% heat-inactivated fetal bovine serum (Gibco, Foster City, CA) and 1\% penicillin-streptomycin solutions (Gibco) in a humidified incubator with $5 \% \mathrm{CO}_{2}$ at $37^{\circ} \mathrm{C}$. The enteric neurons were cultured in Dulbecco's modified Eagle's medium and observed their growth every day. The next day, we added cytarabine (Gibco, $5 \mu \mathrm{M}$ ) to culture to inhibit the growth of glial cells and miscellaneous cells, and then pure cultured primary neurons were obtained. The macrophages were irritated with LPS $(100 \mathrm{ng} / \mathrm{ml})$ for $6-8 \mathrm{~h}$ or IL-4 $(10 \mathrm{ng} / \mathrm{ml})$ for $24 \mathrm{~h}$ before collecting different macrophages supernatant. Next, the enteric neurons were handled with different macrophages supernatant for $24 \mathrm{~h}$.

\subsection{Experimental animals}

Wild type C57BL6/J mice were bought from Beijing Vital River Laboratory Animal Technology and housed in a specific pathogen-free environment in the Animal Center of Shandong University. All mice in the experiment were male and aged 7-10 weeks and were randomly grouped. All experiments were operated at the Animal Center of Shandong and approved by the Medical Ethics Committee for Experimental Animals, Shandong University School of Basic Medicine Sciences (ECAESDUSM 2014056). Certainly, mice suffering was minimized.

\subsection{Immunocytofluorescense}

The paraffin sections of mice colon were dewaxed for $2 \mathrm{~h}$, which were soaked in two xylenes for 5 min and soaked with 100\% alcohol, $95 \%$ alcohol, $90 \%$ alcohol, $75 \%$ alcohol, 50\% alcohol for $3 \mathrm{~min}$, and then rinsed with Water for $3 \mathrm{~min}$. Then, repaired by boiling slices in sodium citrate buffer $(10 \mathrm{mM}, \mathrm{PH}=6$, Beyotime) for $25 \mathrm{~min}$. After cooling down, tissues were blocked with $10 \%$ donkey serum for 50 min at $25^{\circ} \mathrm{C}$ and then incubated with rabbit anti-F4-80 (1:200, Abcam) and anti-iNOS (1:200, Abcam) primary antibodies diluted by blocking solution overnight at $4{ }^{\circ} \mathrm{C}$. After three washes, the slides were incubated with Alexa Fluor 568 or 488 donkey anti-rabbit (1:1000, Invitrogen) or Alexa Fluor 488 donkey anti-mice (1:1000, Invitrogen) secondary antibodies for 60 min and then counterstained with DAPI (1:1000, Beyotime) for $5 \mathrm{~min}$. The images were Caught by a fluorescent microscope (Olympus IX71).

\subsection{RNA extraction and quantitative real-time PCR}

The total RNA was extracted from neurons and LMMP by a tissue/cell rapid extraction kit (Spark jade, Qingdao, China). In short, Lysis Solution RL was added to lyse cells and tissue fluid after homogenization in order to depolymerize and release protein and nucleic acid substances. Next, chloroform, absolute ethanol, protein-removing solution RE and rinsing solution RW were added in sequence to obtain about 
$20 \mu$ l of RNA solution. RNA was reversed transcribed employing a Takara PCR Thermal Cycler SP (Takara Bio, Shiga, Japan). Real-time quantitative PCR was carried through with the SYBR Premix Dimer Eraser (Takara Bio, Shiga, Japan). Expression levels were normalized to the internal controls ( $\beta$-actin) and the relative expression level was evaluated using the $2^{-} \triangle \triangle \mathrm{CT}$ method. The primers were designed and verified by Shanghai Genomics Institute (Shanghai, China) and the sequences of the primers used were demonstrated in Table 1.

\subsection{ELISA}

To test the concentration of IL-1 $\beta$, TNF- $\alpha$, IL- 6, TGF- $\beta$, OT, the enteric neurons and tissues supernatant were gathered and centrifugalized after handled, the concentrations of a specific protein were measured using a pre-coated ELISA kit according to the instruction manual.

\subsection{Culture of colon explant}

Colons from naive or colitis mice were flushed 10 times with PBS to remove faces. Two 5-mm-long colon tissues were cultured in $250 \mu$ l of

$\mathrm{RPMl} / 10 \% \mathrm{FCS} / 1 \%$ penicillin-streptomycin/50 mg/ml 1gentamycin (Wako) in a 48-well plate (Corning, $\mathrm{NY}$ ) at $37^{\circ} \mathrm{C}$ for $30 \mathrm{~min}$ [23]. OT in the culture supernatant was measured by ELISA kit.

\subsection{Western blotting analysis}

Enteric neurons and tissues were gathered into ice-cold RIPA buffer (Bioster Bio, Pleasanton, CA) including $1 \%$ PMSF and $1 \%$ phosphatase inhibitor (Bioster Bio, Pleasanton, $\mathrm{CA}$ ) after stimulation with specific drugs, the cells were centrifuged at $13800 \times \mathrm{g} / 10 \mathrm{~min}$ at $4^{\circ} \mathrm{C}$. Keeping the supernatant and the BCA protein assay kit (Boster, USA) was used to quantify total protein concentration. Next, the samples were blended with $5 \times$ loading buffer and boiled up for $10 \mathrm{~min}$ at $100^{\circ} \mathrm{C}$. Then the proteins were loaded onto a $15 \%, 10 \%$ and $8 \%$ gradient polyacrylamide gel, electrophoretically transferred to polyvinylidene difluoride membrane, the membranes were blocked with $5 \%$ non-fat dry milk for 90 min at $25^{\circ} \mathrm{C}$. The membranes were handled with the following primary antibodies, anti-OT (1:400, Invitrogen) antibody, antiOTR (1:800, Abcam) antibody, anti-PEG3(1:800) antibody, anti-p65 (1:1000, CST) antibody, anti-phosphop65 (1:800, CST) antibody, anti-STAT3 (1:1000, CST) antibody, anti-phospho-STAT3 (1:1000, CST) antibody, phospho-SMAD2 $(1: 400, C S T)$ antibody, anti-SMAD2 (1:1000,CST) antibody, phosphoSMAD3(1:500,CST) antibody, anti-SMAD3 (1:1000,CST) antibody and anti- $\beta$-actin (1:2000,Zhongshan Golden Bridge Biotechnology) antibody, overnight at $4^{\circ} \mathrm{C}$. After multiple washes, membranes were incubated with secondary antibodies (1:2000, Beyotime) at room temperature for $50 \mathrm{~min}$. The ECL kit reagent (MILLIPORE, USA) was used to generate chemiluminescence signal, which were caught with the Tanon Imaging System (Tanon-4600). The signal strength was collected by ImageJ software.

\subsection{DSS colitis model}

Acute colitis was induced by density of 2.5\% DSS (Dextran Sulfate Sodium) salt (reagent-grade, MW 36$50 \mathrm{kDa}$, MP Biomedicals, Canada) in the drinking water for 7 days, then the mice started drinking water 
from the second week. The Mice were randomly allocated into six groups: (1) Control (2) 1W-DSS (3) 1WDSS + 1W-WATER (4) 1W-DSS + 2W-WATER (5) 1W-DSS + 3W-WATER (6) 1W-DSS + 6W-WATER. Mice were euthanized by intraperitoneal administration of sodium pentobarbital $(200 \mathrm{mg} / \mathrm{kg})$ on the day 7,14 , 21, 28 and 49. Distal colon was embedded with $4 \%$ paraformaldehyde solution, and the transverse sections were stained with hematoxylin and eosin. Two analysts were made to measure the histological scores, blinded to each animal groups. The assessment criteria incorporated epithelial surface damage, the loss of crypts and inflammatory infiltrate described by Zaki MH, ranging from 0 to 6 (combining inflammatory cells infiltration score and tissue damage score).

\subsection{D-mannose model}

The mice were fed 20\% D- mannose (Sigma-Aldrich, St. Louis, MO) for 7 days [24, 25]. Clodronate liposomes (FormuMax, Silicon Valley, CA) were injected intraperitoneally one day before the start of the model and the third day after the model to deplete macrophages, and the control liposomes were used as the control group[26, 27]. The Mice were randomly allocated into six groups: (1) Control, (2) Control + Dmannose, (3) Control Liposomes, (4) Control Liposomes + D-mannose, (5) Clodronate Liposomes, (6) Clodronate Liposomes +D-mannose. Distal colons were embedded with $4 \%$ paraformaldehyde solution, and the transverse sections were stained with hematoxylin and eosin.

\section{Statistical analysis}

Data was expressed as mean $\pm S E M$ from at least two independent experiments, and presented the number of samples in the specific experiments. One-way ANOVA or two-tailed Student's $t$-test were used to compare between groups. GraphPad Prism version 5 (La Jolla, CA) was used for statistical analysis. $P$ $<0.05$ was considered statistically significant.

\section{Results}

\subsection{Effect of macrophages polarization on OT signaling system}

To investigate the effect of macrophages polarization on the OT signaling system, we first isolated and extracted enteric neurons (Figure S1A), then, we used LPS or IL-4 to stimulate enteric neurons, and found that OT or OTR mRNA did not evidently change compared to the control group and the secretion of OT did not change (Fig. 1A, B, C). Next, we used LPS to induce RAW264.7 cells to M1 type and IL-4 to M2 type, and collected no-stimulated macrophages supernatant (M0 supernatant), LPS-stimulated macrophages supernatant (M1 supernatant), IL4-stimulated macrophages supernatant (M2 supernatant), added them to the culture fluid of the enteric neurons (Fig. 1D). We found that after the treatment of M1 supernatant, the levels of OT or OTR mRNA were markedly dropped compared with the control group (Fig. 1E), while treatment of M2 supernatant increased the expression of OT or OTR mRNA (Fig. 1F). At the same time, we found that macrophages supernatant had similar effect on the expression of OT and OTR proteins (Fig. 1G, H, I). 
3.2. Effect of cytokines in macrophages supernatant on the expression of OT and OTR in enteric neurons

In order to investigate cytokines in M1 or M2 macrophages supernatant that regulated the expression OT and OTR, firstly, we measured the levels of different cytokines in the supernatant. We found that in M1 supernatant, the levels of TNF- $a$, IL- 6 and IL-1 $\beta$ were obviously increased (Fig. 2A, B, C) while in M2 supernatant, the level of TGF- $\beta$ was obviously increased (Fig. 2D). Secondly, we tested the effects of these cytokines on the expression of OT and OTR mRNA. We found that treatment of IL-6, TNF- $a$ or IL-1 $\beta$ significantly decreased the expression of OT and OTR mRNA in cultured enteric neurons (Fig. 2E, F, G), and reduced the release of OT in cultured enteric neurons (Fig. 2J). On the contrary, TGF- $\beta$ increased the levels of OT and OTR mRNA in cultured enteric neurons (Fig. 2I), but IL-10 seemed to have no effect (Fig. 2H).

In order to testify whether the cytokines affected the expression of OT and OTR through their receptors, we treatment the cultured enteric neurons with the receptor antagonists. We found that IL-6 receptor antagonist tocilizumab, TNF-a receptor antagonist R-7050, and TGF- $\beta$ antagonist LY2109761 reversed the inhibitory (IL- 6 and TNF- $\alpha$ ) and excitatory (TGF- $\beta$ ) effect of the respective agonists (Fig. 3A, B, C, Figure S1B). Besides this, we also found that treatment of IL-1Ra, tocilizumab and R-7050 completely reversed the excitatory effect of M1 supernatant on the expression of OT, and LY2109761 completely reversed the inhibitory effect of M2 supernatant (Fig. 3D, E). So, we believed that the inhibitory effect of M1 supernatant on OT and OTR expression was mainly through IL-1 $\beta$, IL-6 and TNF-a, and the excitatory effect of M2 supernatant was mainly through TGF- $\beta$.

\subsection{Inflammatory cytokines downregulated expression of OT/OTR proteins in enteric neurons via STAT3 or NF-kB pathway}

It has been reported that inflammatory factors could activate STAT3 [28] and NF-KB pathways to participate in intracellular reactions $[20,29]$. In this study, we investigated whether these two pathways were involved in the downregulation the expression of OT and OTR proteins following IL-6, TNF-a and IL$1 \beta$ treatment. The results showed that degree of STAT3 phosphorylation was significantly higher compared to the control group following treatment of IL-6 or TNF-a for $24 \mathrm{~h}$ (Fig. 4A, C, E, G). At the same time, the expression of OT and OTR proteins decreased significantly, and this inhibitory effect was reversed by stattic (STAT3 inhibitor, $5 \mu \mathrm{M}$ ) (Fig. 4B, D, F, H). Treatment of IL-1 $\beta$ did not change the degree of STAT3 phosphorylation (Figure S1C) but significantly up-regulated the expression of p-NF-kB protein in enteric neurons (Fig. $4 \mathrm{I}, \mathrm{K}$ ). The decrease of OT and OTR expression following IL-1 $\beta$ administration was significantly reversed by pretreatment of Bay11-7082 (NF-KB inhibitor, $1 \mu \mathrm{M})$ (Fig. 4J, L). Therefore, we believed that the inflammatory cytokines in M1 macrophages supernatant inhibited the expression of OT and OTR in different pathways, TNF-a and IL- 6 might through the STAT3 phosphorylation, while IL-1 $\beta$ might mainly through NF-KB phosphorylation. 


\subsection{Smad2/3 pathway was involved in the upregulation of OT/OTR expression following TGF- $\beta$}

TGF- $\beta$ induces cellular response by binding transmembrane receptors [30] and phosphorylation of Smad2/3 (receptor regulated Smads: R-Smads) [31]. In this study, we found that the expression of $p$ Smad 2 and $p$-Smad 3 were up-regulated following TGF- $\beta$ treatment for $24 \mathrm{~h}$, and pretreatment of SIS $3 \mathrm{CHL}$ (Smad3 inhibitor, $5 \mu \mathrm{M}$ ) blocked this change (Fig. 5A, B, C, D). So, we believed that TGF- $\beta$ increased the OT and OTR expression in enteric neurons via Smad2/3 pathway.

\subsection{Correlationship between macrophages polarization and expression of OT and OTR in DSS-induced colitis}

Previous studies in our laboratory have shown that the OT signaling system reduced intestinal inflammation by regulating the polarization of macrophages in experimental colitis [7, 20,32]. In this study, we explored whether the expression of OT signaling system was affected in DSS induced colitis (Figure S2A). In the first 7 days during which the mice were fed with DSS, we found that compared with the control group, the mice in the DSS treatment group had significantly reduced body weight, reduced colon length, and a higher disease activity index (Figure S2B, C, D, E). These changes reached the maximal level at the end of the first week after the beginning of the DSS feeding and then began to decrease. At the third week, the weight of the mice and the colon length returned to normal, and the disease activity index decreased significantly (Fig. 2SB, C, D, E). Histological evaluation at different time periods showed that, at the 7day following DSS treatment, the colon appeared severe epithelial damage, crypt loss, submucosal edema, and inflammatory cell infiltration. However, these histological changes began to decrease at the second week and completely disappear at the seventh week (Figure S2F, G). So, we divided DSS-induced colitis into inflammatory phases (the first and second week) and recovery phases (the third week later) (Figure S2A). Previous data indicated that M1 supernatant and IL-1 $\beta$, TNF- $a$, IL- 6 inhibited the levels of OT and OTR mRNA expression in cultured enteric neurons, while M2 supernatant and TGF- $\beta$ promoted them (Fig. 1, 2). In the inflammatory phase of DSS-induced colitis, the mRNA expression of IL-1 $\beta$, TNF- $\alpha$ and IL-6 in colon were significantly increased (Fig. 6A, B, C), the mRNA expression of M1 macrophages markers CCL2 and iNOS (Fig. 6D), and the numbers of F4-80/iNOS double positive cells were also significantly increased (Figure S3A). They reached the highest level at the first or second week, and gradually decreased to normal in the recovery period. On the other hand, the mRNA expression of TGF- $\beta$, and M2 macrophages markers CD206, Arg1, Chil3 did not change at the first week, but began to increase at the second week and reached the highest level at the third or fourth week (Fig. 6E, F, G). The concentration of IL-6, IL-1 $\beta$, TNF- $\alpha$ and TGF- $\beta$ showed a similar dynamic change (Fig. $6 \mathrm{H}, \mathrm{I})$. Therefore, we believed that macrophages were polarized to $\mathrm{M} 1$ type during the inflammatory phase and to $\mathrm{M} 2$ type during the recovery phase. Next, we tested the expression of OT signaling system in the two phases in the DSS-treated mice. We found that the expression of OT and OTR mRNA decreased significantly during the inflammatory phases, and increased significantly during the recovery phases (Fig. 6J). The level of OT in colon explant (Fig. 6K) and the density of neurons in the colon (Figure S3B) 
showed the same dynamic changes. we believed that the expression of the OT signaling system and macrophages polarization in colon were correlated at the two phases of DSS induced colitis.

\subsection{M2 macrophages polarization increased the expression of OT and OTR in colon}

In order to further test the hypothesis that M2 macrophages polarization increased the OT and OTR expression in the gut, we used D-mannose (a commonly used glyconutrient in clinical practice) to activate macrophages [33]. The mice were fed D-mannose for 7 days, the body weight decreased on the second day and began to recover on the fourth day (Figure S4A). There was no change in the eating habits and histological structure of the colon (Figure S4B, C), but the size and weight of the cecum and colon increased (Figure S4D). It is reported that D-mannose inhibited the M1 polarization [33]. We also found that at 7 days following D-mannose, the iNOS mRNA expression in colon was decreased, but that of IL-6, IL-1 $\beta$ and TNF-a did not change (Figure S4F). On the other hand, to our surprise, we found that the mRNA expression of surface markers of M2 macrophages in colon, including CD206, Arg1, Ym1 and Chil3 were significantly increased, and the anti-inflammatory factor TGF- $\beta$ and IL10 were also significantly upregulated. So, we believed that D-mannose promoted polarization of M2 macrophages and inhibited the polarization of M1 macrophages. Accompanied with the change of the polarization of macrophages, the expression of OT and OTR were also significantly up-regulated (Figure S4G).

In order to prove the hypothesis that the increase of OT signaling system following D-mannose treatment was attributed to the M2 polarization, we applied system administration of clodronate liposomes to deplete macrophages [26,34] (Fig. 7A), and observed that the expression of the OT signaling system. After the clodronate liposomes administration for $24 \mathrm{~h}$, the numbers of $\mathrm{F} 4-80^{+}$cells (Fig. 7E) and the expression of M1 and M2 macrophages surface markers (Fig. 7G) in the colon were significantly reduced. Treatment of clodronate liposomes decreased the body weight, but did not influence the eating and drinking habit, and size and weight of the colon and cecum of the mice (Fig. 7B, C, D, F). Pretreatment of clodronate liposomes significantly reversed the up-regulation of OT and OTR expression in colon induced by systemic administration of D-mannose (Fig. 7H, I). On the other hand, D-mannose itself did not affect the expression of OT and OTR mRNA in the cultured enteric neurons (Figure S4H). These data further proved our hypothesis that M2 macrophages promoted the expression of the OT signaling system in colon.

\subsection{Peg3 was involved in the upregulation of OT and OTR expression induced by M2 macrophages polarization}

Peg3 (Paternally Expressed Gene 3) encodes a DNA binding protein that acts as a inhibitory transcriptional regulator for the expression of OTR [35]. So, we hypothesized that cytokines released from polarized macrophage might influence OT signaling system expression through Peg3. In order to prove this hypothesis, firstly, we tested the expression of Peg3 and OT in the D-mannose treated mice. We found that 7 days following D-mannose treatment, the expression of Peg3 was inhibited, and at the same time, 
the expression of OT mRNA was increased (Fig. 8A, B, C). Because TGF- $\beta$ promoted the expression of the OT signaling system through the Smad2/3 signaling pathway, so we investigated whether the downregulation of Peg3 expression following D-mannose treatment was also through Smad2/3. We found that injection of SIS3CHL, the Smad3 blocker (Fig. 8F), significantly abolished the effect of Dmannose on the expression of Peg3 (Fig. 8D) and OT (Fig. 8E). Secondly, we repeated this experiment in the cultured enteric neurons. We found that treatment of $\mathrm{M} 0$ and $\mathrm{M} 1$ supernatant, and the inflammatory cytokines, including IL-6, IL-1 $\beta$ and TNF- $a$, did not change the expression level of Peg3, but treatment of M2 supernatant and TGF- $\beta$ significantly reduced it compared to the control group in the cultured enteric neurons (Fig. 8G, H). Blocking the Smad3 signaling pathway by $\mathrm{SIS} 3 \mathrm{HCl}$ significantly attenuated the downregulation of Peg3 expression and upregulation of OT and OTR expression (Fig. 8I). These results indicated that M2 macrophages and TGF- $\beta$ increased OT and OTR expression through downregulation of Peg3 expression.

\section{Discussion}

Our current study demonstrated that the polarization of macrophages affected the expression of OT signaling system in the gut. In vitro, M1 macrophages inhibited the expression of the OT signaling system through secretion of pro-inflammatory factors, including IL-1 $\beta$, IL- 6 and TNF- $a$, and the intracellular STAT3, NF-KB pathways in enteric neurons. On the other hand, M2 macrophages promoted the expression of the OT signaling system in enteric neurons through secretion of TGF- $\beta$ and the intracellular Smad2/3Peg3 pathway. This is the first time to report that macrophages polarization differentially regulated the OT and OTR expression in enteric neurons.

OT is a stress hormone, which changes during infection and inflammation [36-38]. As we know, in response to LPS stimulation, the recruited M1 macrophages produced large amounts of pro-inflammatory cytokines, such as IL-1 $\beta$, IL-6, TNF-a. IL-4 treatment induced the M2 macrophages polarization and released anti-inflammatory cytokines such as TGF- $\beta$ and IL-10 [39]. We found that the pro-inflammatory cytokines, including IL-1 $\beta$, IL- 6 and TNF-a inhibited OT and OTR expression in enteric neurons, while TGF$\beta$ exerted an excitatory effect. This data was consistent with the observation that $\mathrm{M} 1$ supernatant inhibited the OT and OTR expression and M2 supernatant increased it. So, we thought the cytokines in the M1 supernatant that inhibited the OT and OTR expression in enteric neurons might be IL-1 $\beta$, IL-6 and TNF- $a$, and the cytokines in M2 supernatant that increased OT and OTR expression might be TGF- $\beta$. Moreover, pretreatment of the antagonists of these cytokines abolished the effect of M1 and M2 supernatant. So, we believed that following activation, the M1 macrophages decreased the OT and OTR expression in enteric neurons through pro-inflammatory cytokines IL-1 $\beta$, IL- 6 and TNF- $a$, and M2 macrophages increased them through anti-inflammatory cytokine TGF- $\beta$. After these inflammatory factors bound to their receptors, then they participated in biological processes through a series of signaling pathways such as JAK-STAT3 and NF-KB [28, 29, 40, 41]. In agreement with the above observations, our results showed that the pro-inflammatory factors IL- 6 and TNF-a activated STAT3 signaling pathway, and IL-1 $\beta$ activated NF-KB signaling pathway to inhibit the expression of OT and OTR. The data of the experiments that STAT3/NF-KB signaling pathway were blocked further prove these 
results. One of the interesting finding of the present study was that M2 polarized macrophages increased the expression of OT and OTR in enteric neurons, which might be mediated by TGF- $\beta$. TGF- $\beta$ mainly regulated downstream cell responses through Smad and non-Smad signaling pathways [31, 42]. Our experiments confirmed that TGF- $\beta$ activated Smad2/3 and promoted the expression of OT and OTR. Both LY2109761(TGF- $\beta$ receptor inhibitor) and SIS3HCL (Smad3 blocker) completely abolished the excitatory effect of TGF- $\beta$ on the excitatory effect of OT and OTR. So, we believed that TGF- $\beta$ upregulated the expression of OT and OTR in enteric neurons mainly through intracellular Smad2/3 pathway.

In order to confirm the correlation between macrophages polarization and OT/OTR expression in vivo, we used DSS induced colitis and investigated the temporal relationship between the inflammation and OT/OTR expression. We found that the disease activity index and the levels of pro-inflammatory cytokines in the colon increased and reached the highest level at the second week, so we defined this period as the inflammatory phase. In this period, correlated with the activation of M1 macrophages and the increased of the pro-inflammatory cytokines, the expression of OT and OTR in ENS were also decreased. Three weeks later after the DSS administration, the disease activity of the mice gradually returned to normal, and the expression of M2 macrophages markers and the anti-inflammatory cytokine began to increase and reached to the highest level at fourth week and returned to normal at the fifth to seventh week. We defined this period as the recovery period. In this period, correlated with the polarization of macrophages to M2 type and the release of TGF- $\beta$, the expression of OT/OTR in ENS was also increased. This observation further supported our hypothesis that following the acute inflammation, there were dynamic changes of the OT/OTR expression correlated to the different polarization of the macrophages in gut. The M1 macrophages polarization correlated to the decrease of OT/OTR expression in inflammatory period, and the M2 macrophages polarization correlated with the increase of OT/OTR expression in the recovery period. As far as we know, this is the first report about the dynamic changes of the OT/OTR expression in ENS following the DSS induced colitis. Although we found that OT inhibited the polarization of macrophages to $\mathrm{M} 1$ type and promoted it to $\mathrm{M} 2$ type, and OTR deficient mice are more susceptible to DSS induced colitis [20,38], the anti-inflammatory effect of OT might not be very important at the acute inflammatory period, because during this period, with the release of pro-inflammatory cytokines from M1 macrophages, the expression of OT and OTR in ENS was decreased. But on the other hand, it was interesting to find that the OT/OTR expression was increased during the recovery period, which might be promoted by the polarization of M2 macrophages and the released TGF- $\beta$. During the recovery period of the acute inflammation, the function of the polarization of $\mathrm{M} 2$ macrophages and the released anti-inflammatory cytokines were mainly to confine the inflammation and promote the proliferation of the damaged tissue[12, 23, 43-45]. During this period, there was a positive feedback between the M2 macrophages activity and expression of OT and OTR in ENS, so it was possible that OT signaling system in ENS was involved in tissue repair following the local acute inflammation in gut.

D-mannose prevented acute lung injury by regulating PPAR- $\gamma$ and TGF- $\beta$ level [24], and inhibited LPSinduced macrophages activation by impairing IL-1 $\beta$ production. In vivo, D-mannose improved endotoxemia and relieve colitis [33]. Therefore, we used D-mannose to induce polarization of M2 macrophages in the mice. We found that following D-mannose administration, the expression iNOS in gut 
were significantly down-regulated, but TGF- $\beta$, CD206, Arg1 were significantly increased. The expression of OT and OTR in colon was also increased. The above data indicated that D-mannose inhibited the polarization of M1 macrophages and promoted the polarization of M2 macrophages. The increase of OT and OTR expression following treatment of D-mannose might be caused by the polarization of M2 macrophages. In order to further testify this hypothesis, we used clodronate liposomes to deplete macrophages in vivo. The upregulation of OT and OTR expression was abolished by the depletion of the macrophages. So, we believed that, in vivo, D-mannose increased the OT and OTR expression in ENS through polarization of macrophages to $\mathrm{M} 2$ type. Another interesting finding in these experiments was that, following D-mannose administration, the size and weight of the gut were increased, although the histological structure of the intestine did not change. This change was macrophages dependent. Therefore, we thought that it might be caused by the cytokines such as TGF- $\beta$ released from polarized M2 macrophages and OT released from the ENS.

As is known, Peg3 is widely expressed in various tissues of humans and mice, encodes a DNA-binding protein involved in the control of specific target genes of different cell functions [46] and plays a role as a transcriptional regulator for the expression of OTR. In Peg3-KO mice, the expression of OTR in hypothalamus and breast were up-regulated [35]. In this study, we first proved that M2 supernatant and TGF- $\beta$ promoted the expression of OT by suppressing the expression of peg 3 in enteric neurons. But the M0/M1 macrophages and pro-inflammatory factors did not affect the expression of Peg3.

Notably, many reports indicated that the ENS cooperated with intestinal macrophages to maintain intestinal immune homeostasis $[47,48]$. The unique location between mucosal macrophages and enteric neurons has attracted great attention from researchers [49]. Emerging data suggested that MMs secreted cytokines to protect nerves from damage and promote nerve regeneration [50]. These might be the results of the interaction between the ENS and the immune system. In this study, we found that, polarization of macrophages affected expression of enteric neurons OT signaling system. In the recent study, we reported that OT signaling system regulated the polarization of macrophages. This "feedback loop" between macrophages and enteric neurons could be a pivotal "controller" that decided whether the organism tends to be homeostasis or leads to disease.

\section{Conclusion}

In summary, the present findings demonstrate that the polarization of macrophages affected the expression of OT signaling system in the gut. In vitro, M1 macrophages inhibited the expression of the OT signaling system through secretion of pro-inflammatory factors, including IL-1 $\beta$, IL- 6 and TNF-a, and the intracellular STAT3, NF-KB pathways in enteric neurons. On the other hand, M2 macrophages promoted the expression of the OT signaling system in enteric neurons through secretion of TGF- $\beta$ and the intracellular Smad2/3-Peg3 pathway. The OT signaling system may also be a potential drug target for the treatment of intestinal diseases caused by abnormal immune systems.

\section{Abbreviations}


ENS, enteric nervous system; LMMP, longitudinal muscle myenteric plexus; OT, oxytocin; OTR, oxytocin receptor; DAPI, 4',6' diamidino-2-phenylindole dihydrochloride hydrate; FBS, fetal bovine serum; IHC, immunohistochemistry; LPS, lipopolysaccharide; qRT-PCR, reverse transcriptase quantitative real-time PCR; MMs, muscularis macrophages; IL, interleukin; TNF- $\alpha$, tumor necrosis factor- $\alpha$; TGF- $\beta$, Transforming growth factor $\beta$; Arg1, arginase1; STAT3, Signal transducer and activator of transcription3; Chil3, chitinase-like 3; DSS, dextran sulfate sodium; D-M, D-mannose; IBD, inflammatory bowel disease; iNOS, inducible nitric oxide synthase.

\section{Declarations}

\section{Acknowledgements}

We thank the animal medicine center of Shandong University for providing experimental animals and the Basic Medical Sciences of Shandong University for providing experimental places and basic materials.

\section{Availability of data and materials}

The data generated during our study cannot be publicly available due to the data safety concern. But the data are available from the corresponding author on reasonable request

\section{Funding}

This work was supported by grants from the National Scientific Foundation of China [grant numbers NSFC31871160 and 32071127].

\section{Author Contributions}

CYL: made substantial contributions to study design, data interpretation, writing and revising of the manuscript, and final revision of the manuscript; YS: made substantial contributions to the laboratory work, analyses of data and editing of the manuscript; SL and HJZ: performed animal model; JCZ and TTC performed cells culture; BY and JXL: manuscript proof reading and editing; All authors read and approved the final manuscript.

\section{Declaration of Competing Interest}

The authors declare no conflict of interest.

\section{Ethics approval and consent to participate}

We conducted animal experiments according to International Guiding

Principle for Animal Research that were stipulated by the Council for

International Organizations of Medical Sciences (CIOMS), the routines have obtained approval from Shandong University Animal Ethics Committee. The participants working with the animal model received 
training abiding by the rules of Institutional Animal Care and Use Committee Guidebook (IACUC). And written informed consent was obtained from all participants.

\section{Consent for publication}

Not applicable. This article does not contain any individual person's data.

\section{Competing interests}

The authors declare that they have no competing interests.

\section{References}

1. Sala NL, et al. Oxytocin reproducing reflex milk ejection in lactating women. J Appl Physiol. 1974;36(2):154-8.

2. Gimpl G, Fahrenholz F. The Oxytocin Receptor System: Structure, function, and regulation. Physiol Rev. 2001;81(2):629-83.

3. Jurek B, Neumann ID. The Oxytocin Receptor: From Intracellular Signaling to Behavior. Physiol Rev. 2018;98(3):1805-908.

4. Yang HP, et al. Nonsocial functions of hypothalamic oxytocin. ISRN Neurosci. 2013;2013:179272.

5. Jin D, et al. CD38 is critical for social behaviour by regulating oxytocin secretion. Nature. 2007;446(7131):41-5.

6. Che T, et al. Oxytocin hyperpolarizes cultured duodenum myenteric intrinsic primary afferent neurons by opening BK(Ca) channels through IP(3) pathway. J Neurochem. 2012;121(4):516-25.

7. Chen D, et al. Oxytocin evokes a pulsatile PGE2 release from ileum mucosa and is required for repair of intestinal epithelium after injury. Sci Rep. 2015;5:11731.

8. Gong L, et al. The antinociception of oxytocin on colonic hypersensitivity in rats was mediated by inhibition of mast cell degranulation via Ca(2+)-NOS pathway. Sci Rep. 2016;6:31452.

9. Kim YS, et al. Priming of mesenchymal stem cells with oxytocin enhances the cardiac repair in ischemia/reperfusion injury. Cells Tissues Organs. 2012;195(5):428-42.

10. Reichardt $F$, et al. Western diet induces colonic nitrergic myenteric neuropathy and dysmotility in mice via saturated fatty acid- and lipopolysaccharide-induced TLR4 signalling. J Physiol. 2017;595(5):1831-46.

11. Mikkelsen HB. Macrophages in the external muscle layers of mammalian intestines. Histol Histopathol. 1995;10(3):719-36.

12. Zhu W, et al. Disequilibrium of M1 and M2 macrophages correlates with the development of experimental inflammatory bowel diseases. Immunol Invest. 2014;43(7):638-52.

13. Ai L, et al., Synbindin restrains proinflammatory macrophage activation against microbiota and mucosal inflammation during colitis. Gut, 2021. 
14. Phillips RJ, Powley TL. Macrophages associated with the intrinsic and extrinsic autonomic innervation of the rat gastrointestinal tract. Auton Neurosci. 2012;169(1):12-27.

15. Matteoli G, et al. A distinct vagal anti-inflammatory pathway modulates intestinal muscularis resident macrophages independent of the spleen. Gut. 2014;63(6):938-48.

16. Meroni E, et al. Intestinal macrophages and their interaction with the enteric nervous system in health and inflammatory bowel disease. Acta Physiol (Oxf). 2019;225(3):e13163.

17. Muller PA, et al. Crosstalk between muscularis macrophages and enteric neurons regulates gastrointestinal motility. Cell. 2014;158(2):300-13.

18. Hoover DB. Cholinergic modulation of the immune system presents new approaches for treating inflammation. Pharmacol Ther. 2017;179:1-16.

19. Matheis F, et al. Adrenergic Signaling in Muscularis Macrophages Limits Infection-Induced Neuronal Loss. Cell. 2020;180(1):64-78 e16.

20. Tang $Y$, et al. Oxytocin system alleviates intestinal inflammation by regulating macrophages polarization in experimental colitis. Clin Sci (Lond). 2019;133(18):1977-92.

21. Welch MG, et al. Expression and developmental regulation of oxytocin (OT) and oxytocin receptors (OTR) in the enteric nervous system (ENS) and intestinal epithelium. J Comp Neurol. 2009;512(2):256-70.

22. Lourenssen S, Miller KG, Blennerhassett MG. Discrete responses of myenteric neurons to structural and functional damage by neurotoxins in vitro. Am J Physiol Gastrointest Liver Physiol. 2009;297(1):G228-39.

23. Asano K, et al. Intestinal CD169(+) macrophages initiate mucosal inflammation by secreting CCL8 that recruits inflammatory monocytes. Nat Commun. 2015;6:7802.

24. Zhang D, et al. D-mannose induces regulatory T cells and suppresses immunopathology. Nat Med. 2017;23(9):1036-45.

25. Jokela TA, et al. Mannose reduces hyaluronan and leukocytes in wound granulation tissue and inhibits migration and hyaluronan-dependent monocyte binding. Wound Repair Regen. 2013;21(2):247-55.

26. Kumamaru $\mathrm{H}$, et al., Liposomal clodronate selectively eliminates microglia from primary astrocyte cultures. Journal of Neuroinflammation, 2012. 9(1).

27. Lalancette-Hebert $M$, et al. Selective ablation of proliferating microglial cells exacerbates ischemic injury in the brain. J Neurosci. 2007;27(10):2596-605.

28. Li B, et al. Increased hepcidin in hemorrhagic plaques correlates with iron-stimulated IL-6/STAT3 pathway activation in macrophages. Biochem Biophys Res Commun. 2019;515(2):394-400.

29. Atreya I, Atreya R, Neurath MF. NF-kappaB in inflammatory bowel disease. J Intern Med. 2008;263(6):591-6.

30. Fransvea $\mathrm{E}$, et al. Blocking transforming growth factor-beta up-regulates E-cadherin and reduces migration and invasion of hepatocellular carcinoma cells. Hepatology. 2008;47(5):1557-66. 
31. Miyake T, Aziz A, McDermott JC. Maintenance of the Undifferentiated State in Myogenic Progenitor Cells by TGFbeta Signaling is Smad Independent and Requires MEK Activation. Int J Mol Sci, 2020. 21(3).

32. Li J, et al. Oxytocin down-regulates mesenteric afferent sensitivity via the enteric OTR/nNOS/NO/KATP pathway in rat. Neurogastroenterol Motil. 2015;27(1):51-62.

33. Torretta S, et al. D-mannose suppresses macrophage IL-1 beta production. Nat Commun. 2020;11(1):6343.

34. Ma Y, et al. Polyethylenimine nanofibrous adsorbent for highly effective removal of anionic dyes from aqueous solution. Science China Materials. 2016;59(1):38-50.

35. Frey WD, et al. Oxytocin receptor is regulated by Peg3. PLoS One. 2018;13(8):e0202476.

36. Friebe-Hoffmann $U$, et al. The influence of interleukin-1beta on oxytocin signalling in primary cells of human decidua. Regul Pept. 2007;142(3):78-85.

37. Matsunaga W, et al. LPS-induced Fos expression in oxytocin and vasopressin neurons of the rat hypothalamus. Brain Res. 2000;858(1):9-18.

38. Dou $D$, et al. Oxytocin signalling in dendritic cells regulates immune tolerance in the intestine and alleviates DSS-induced colitis. Clin Sci (Lond). 2021;135(4):597-611.

39. Gordon S, Martinez FO. Alternative activation of macrophages: mechanism and functions. Immunity. 2010;32(5):593-604.

40. Lawrence T, Natoli G. Transcriptional regulation of macrophage polarization: enabling diversity with identity. Nat Rev Immunol. 2011;11(11):750-61.

41. Taniguchi $\mathrm{K}$, et al. YAP-IL-6ST autoregulatory loop activated on APC loss controls colonic tumorigenesis. Proc Natl Acad Sci U S A. 2017;114(7):1643-8.

42. Huang F, et al. Knockdown of TBRG4 suppresses proliferation, invasion and promotes apoptosis of osteosarcoma cells by downregulating TGF-beta 1 expression and PI3K/AKT signaling pathway. Arch Biochem Biophys. 2020;686:108351.

43. Zhang $Y$, et al. ECM1 is an essential factor for the determination of $M 1$ macrophage polarization in IBD in response to LPS stimulation. Proc Natl Acad Sci U S A. 2020;117(6):3083-92.

44. Moreira TG, et al. CLA-supplemented diet accelerates experimental colorectal cancer by inducing TGF-beta-producing macrophages and T cells. Mucosal Immunol. 2019;12(1):188-99.

45. Wang X, et al. The m6A Reader IGF2BP2 Regulates Macrophage Phenotypic Activation and Inflammatory Diseases by Stabilizing TSC1 and PPARY. Advanced Science; 2021.

46. Thiaville MM, et al. DNA-binding motif and target genes of the imprinted transcription factor PEG3. Gene. 2013;512(2):314-20.

47. Watkins LR, Maier SF. Immune regulation of central nervous system functions: from sickness responses to pathological pain. J Intern Med. 2005;257(2):139-55.

48. Jarret A, et al. Enteric Nervous System-Derived IL-18 Orchestrates Mucosal Barrier Immunity. Cell. 2020;180(1):50-63 e12. 
49. Gabanyi I, et al. Neuro-immune Interactions Drive Tissue Programming in Intestinal Macrophages. Cell. 2016;164(3):378-91.

50. Elabd C, et al. Oxytocin is an age-specific circulating hormone that is necessary for muscle maintenance and regeneration. Nat Commun. 2014;5:4082.

\section{Tables}

Table 1. The primer sequences used in the study as follows.

\begin{tabular}{|c|c|}
\hline Name & Primer sequence $\left(5^{\prime} \rightarrow 3^{\prime}\right)$ \\
\hline \multirow[t]{2}{*}{$\beta$-actin } & F primer: CTATTGGCAACGAGCGGTTCC \\
\hline & R primer: CAGCACTGTGTTGGCATAGAG \\
\hline \multirow[t]{2}{*}{$\mathrm{TNF} \alpha$} & F primer: CCCTCACACTCAGATCATCTTCT \\
\hline & R primer: GCTACGACGTGGGCTACAG \\
\hline \multirow[t]{2}{*}{ IL 6} & F primer: TCCTTCCTACCCCAATTCCA \\
\hline & R primer: GTCTTGGTCCTTAGCCACTCC \\
\hline \multirow[t]{2}{*}{ IL $1 \beta$} & F primer: GGCAACCGTACCTGAACCCA \\
\hline & R primer: CCACGATGACCGACACCACC \\
\hline \multirow[t]{2}{*}{ inOS } & F primer: CCGAAGCAAACATCACATTCA \\
\hline & R primer: GGTCTAAAGGCTCCGGGCT \\
\hline \multirow[t]{2}{*}{$\mathrm{CCL} 2$} & F primer: TTAAAAACCTGGATCGGAACCAA \\
\hline & R primer: GCATTAGCTTCAGTTACGGGT \\
\hline \multirow[t]{2}{*}{ Arg1 } & F primer: TGTCCCTAATGACAGCTCCTT \\
\hline & R primer: GCATCCACCCAAATGACACAT \\
\hline \multirow[t]{2}{*}{$\mathrm{ChIL} 3$} & F primer: GATGGCCTCAACCTGGACTG \\
\hline & R primer: CGTCAATGATTCCTGCTCCT \\
\hline \multirow[t]{2}{*}{ CD206 } & F primer: TGATTACGAGCAGTGGAAGC \\
\hline & R primer: GCTACGACGTGGGCTACAG \\
\hline \multirow[t]{2}{*}{ OTR } & F primer: GGCCGTGTTCCAGGTTCTC \\
\hline & R primer: TGCAAGTATGACCAGACGAC \\
\hline \multirow[t]{2}{*}{ OT } & F primer: ATCACCTACAGCGGATCTCAGAC \\
\hline & R primer: CAGAGCCAGTAAGCCAAGCA \\
\hline \multirow[t]{2}{*}{ IL 10} & F primer: TAACTGCACCCACTTCCCAG \\
\hline & R primer: TTGGCAACCCAAGTAACCCTTA \\
\hline \multirow[t]{2}{*}{ TGF $\beta$} & F primer: AGCTGCGCTTGCAGAGATTA \\
\hline & R primer: AGCCCTGTATTCCGTCTCCT \\
\hline \multirow[t]{2}{*}{ Ym1 } & F primer: AGAAGGGAGTTTCAAACCTGGT \\
\hline & R primer: GTCTTGCTCATGTGTGTAAGTGA \\
\hline
\end{tabular}


A

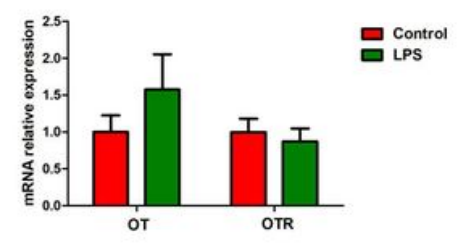

D

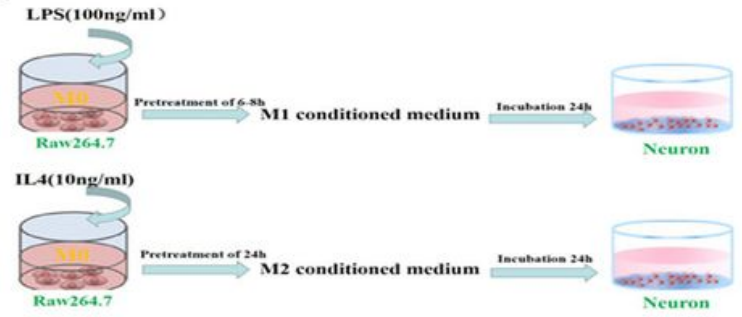

B

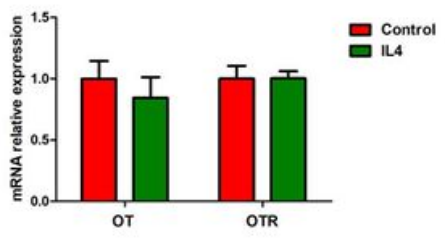

E
C

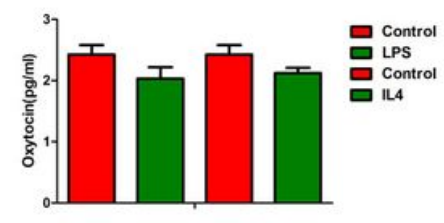

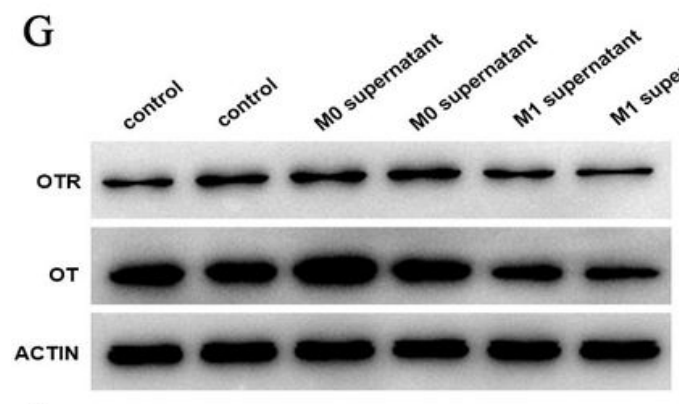

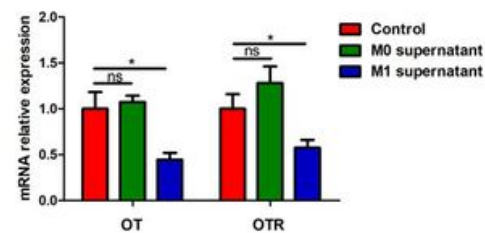

F

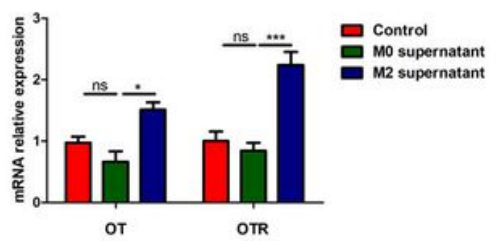

$\mathrm{H}$
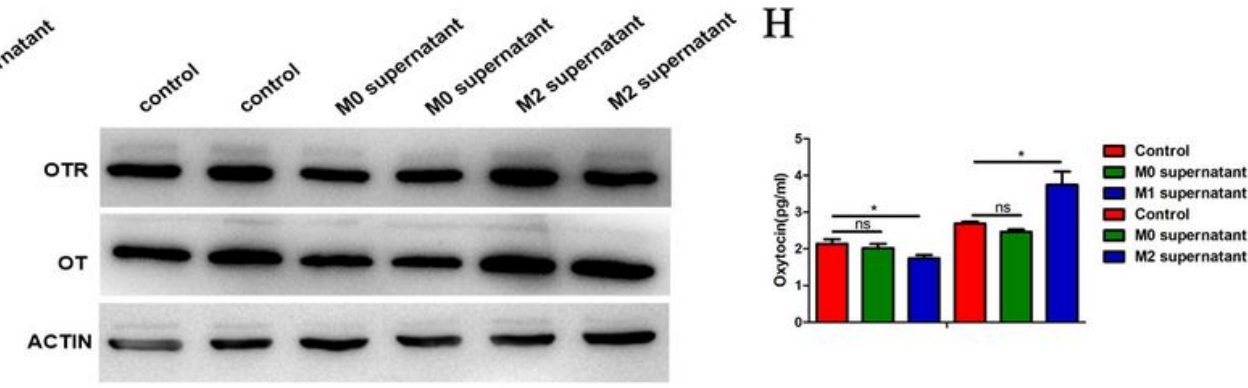

I
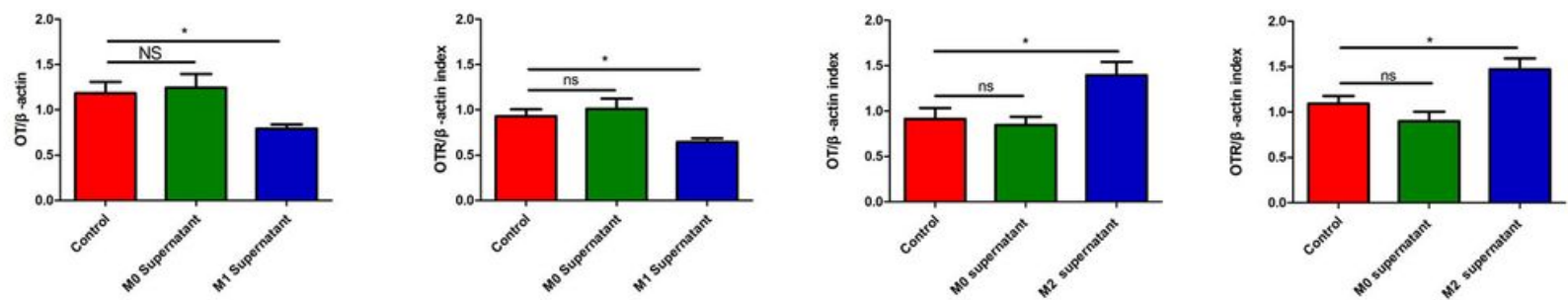

Figure 1

Effect of macrophages polarization on OT signaling system. (A, B) LPS $(100 \mathrm{ng} / \mathrm{ml})$ or IL-4 $(10 \mathrm{ng} / \mathrm{ml})$ stimulated cultured enteric neurons for $24 \mathrm{~h}$ did not affect levels of OT and OTR mRNA. (C) The secretion of OT did not change in cultured enteric neurons with LPS $(100 \mathrm{ng} / \mathrm{ml})$ or IL-4 $(10 \mathrm{ng} / \mathrm{ml})$ treatment for 24 h. (D) Schematic diagram illustrating procedures to obtain conditioned medium. (E, F) The levels of OT and OTR mRNA in cultured enteric neurons were detected with or without conditioned medium treatment for $24 \mathrm{~h}$ via qRT-PCR. M1 supernatant inhibited the levels of OT and OTR mRNA, in contrast M2 supernatant promoted the levels of OT and OTR mRNA. $(G, H, I)$ The levels of OT and OTR proteins in cultured enteric neurons were detected with or without conditioned medium via western blots or ELISA. M1 supernatant inhibited the expression of OT and OTR proteins, in contrast M2 supernatant promoted the expression of OT and OTR proteins. Values represent the mean \pm SEM of 6 samples and were compared by t-test or One-way ANOVA with Dunnett for multiple comparisons. ${ }^{\star} p<0.05$, ${ }^{\star \star \star} p<0.001$ vs control group. 
A

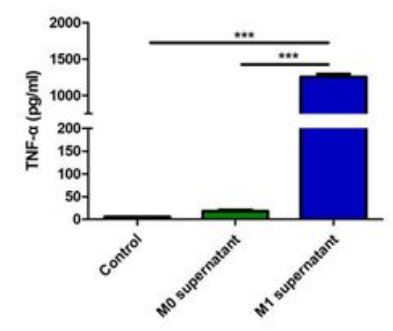

E

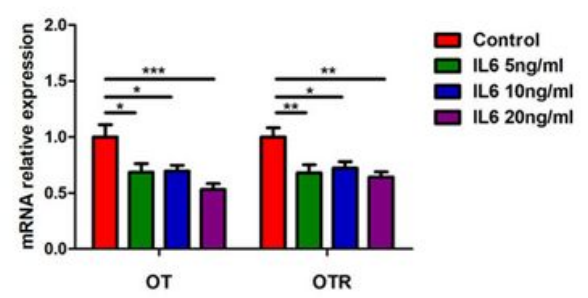

$\mathrm{H}$

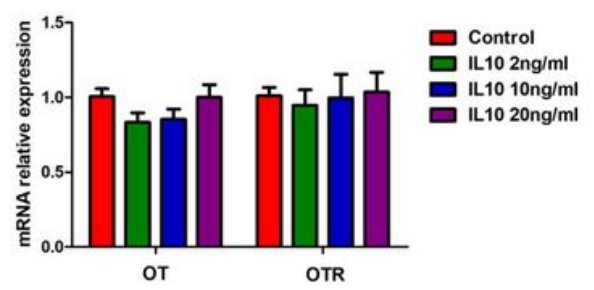

B

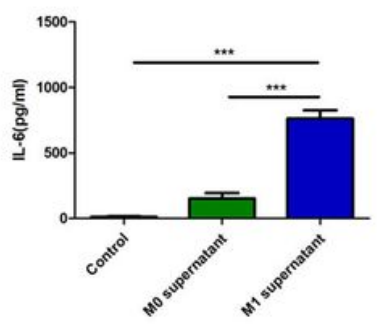

F
C

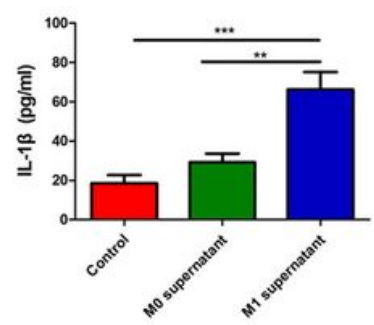

D

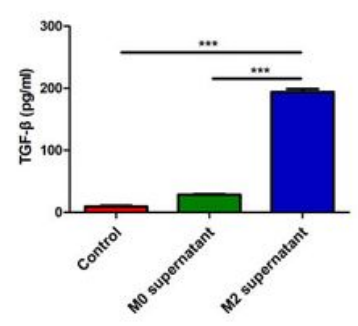

G

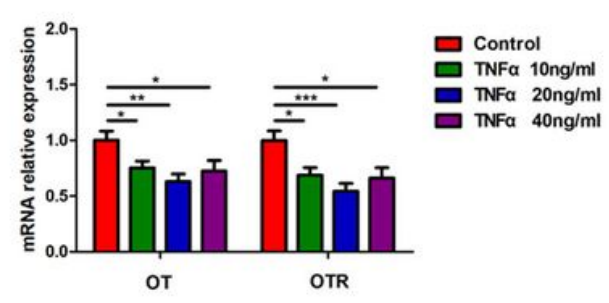

I

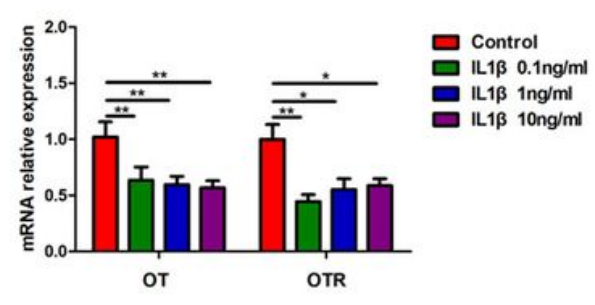

J
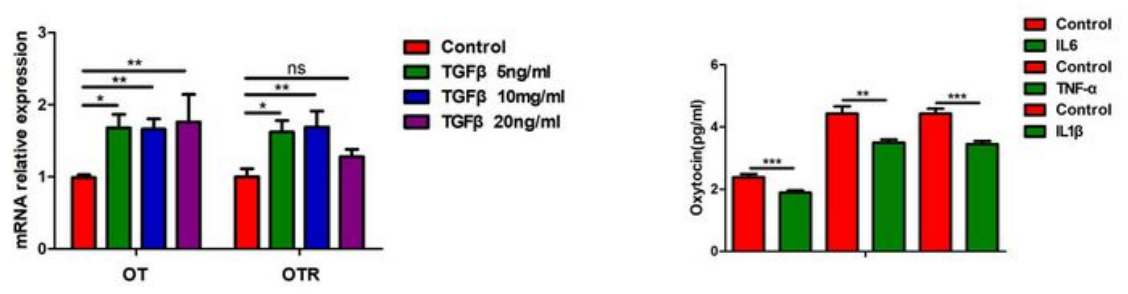

Figure 2

Effect of cytokines in macrophages supernatant on the expression of OT and OTR in enteric neurons. (A, $B, C)$ The levels of IL-6, TNF- $\alpha$ and IL-1 $\beta$ in cultured enteric neurons were increased with M1 supernatant treatment for $24 \mathrm{~h}$ by ELISA. (D) The level of TGF- $\beta$ in cultured enteric neurons was increased with M2 supernatant treatment for $24 \mathrm{~h}$ by ELISA. $(\mathrm{E}, \mathrm{F}, \mathrm{G}) \mathrm{IL}-6(0,5,10,20 \mathrm{ng} / \mathrm{ml})$, TNF- $\mathrm{a}(0,10,20,40 \mathrm{ng} / \mathrm{ml})$ and $\mathrm{IL}-1 \beta(0,0.1,1,10 \mathrm{ng} / \mathrm{ml})$ stimulated cultured enteric neurons for $6 \mathrm{~h}$ decreased the expression of OT and OTR mRNA. (H) IL10 $(0,2,10,20 \mathrm{ng} / \mathrm{ml})$ stimulated cultured enteric neurons did not affect the expression of OT and OTR mRNA. (I) TGF- $\beta(0,5,10,20 \mathrm{ng} / \mathrm{ml})$ stimulated cultured enteric neurons for $6 \mathrm{~h}$ increased the expression of OT and OTR mRNA. (J) The concentration of OT was measured in cultured enteric neurons with or without IL- $6(10 \mathrm{ng} / \mathrm{ml})$, TNF-a $(20 \mathrm{ng} / \mathrm{ml}), \mathrm{IL}-1 \beta(1 \mathrm{ng} / \mathrm{ml})$ treatment for $30 \mathrm{~min}$ by ELISA. Values represent the mean \pm SEM of 6 samples and were compared by One-way ANOVA with NewmanKeuls and Dunnett for multiple comparisons. ${ }^{*} p<0.05$, ${ }^{\star \star} p<0.01$, ${ }^{\star \star *} p<0.001$. 
A

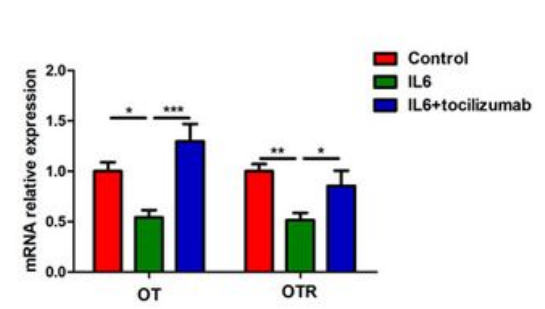

D

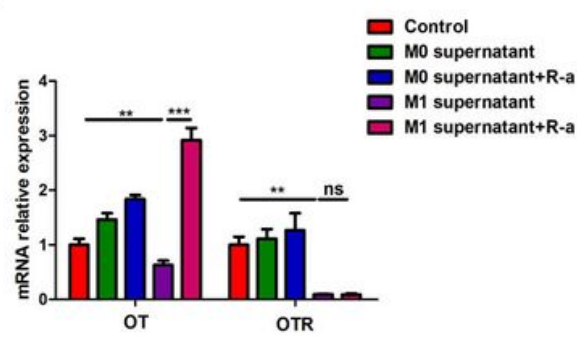

B

$\mathrm{C}$
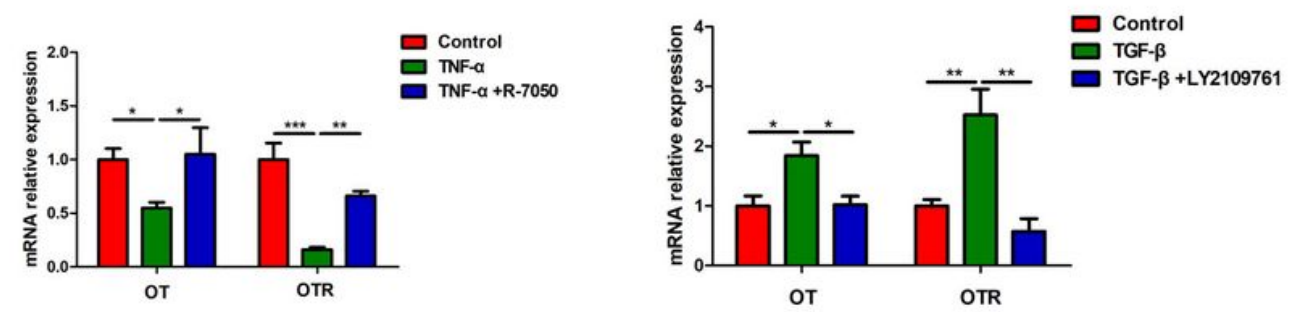

$\mathrm{E}$

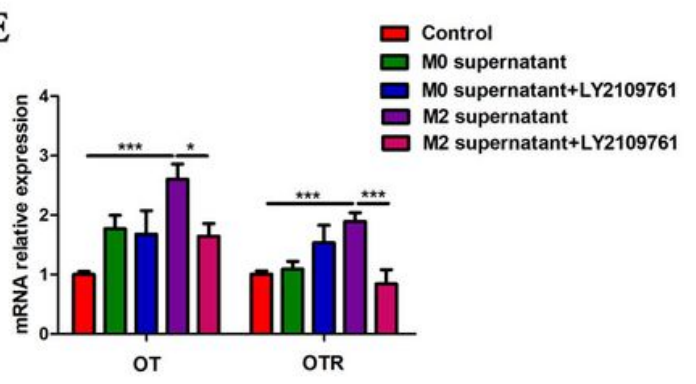

Figure 3

Inhibition of cytokines affected the expression of OT and OTR in cultured enteric neurons. (A) The cultured enteric neurons were pre-treated with tocilizumab (IL6-Ra, $1 \mu \mathrm{M})$ for $12 \mathrm{~h}$ followed by IL-6 (10 $\mathrm{ng} / \mathrm{ml}$ ) for $6 \mathrm{~h}$. The relative expression levels of OT and OTR mRNA were analyzed by qRT-PCR. (B) The cultured enteric neurons were pre-treated with R-7050 (TNF-a receptor inhibitor, $1 \mu \mathrm{M}$ ) for $12 \mathrm{~h}$ followed by TNF-a $(20 \mathrm{ng} / \mathrm{ml})$ for $6 \mathrm{~h}$. The relative expression levels of OT and OTR mRNA were analyzed by qRT-PCR. (C) The cultured enteric neurons were pre-treated with LY2109761 (TGF- $\beta$ receptor inhibitor, $1 \mu \mathrm{M}$ ) for $12 \mathrm{~h}$ followed by TGF- $\beta$ (10 $\mathrm{ng} / \mathrm{ml})$ for $6 \mathrm{~h}$. The relative expression levels of OT and OTR mRNA were analyzed by qRT-PCR. (D) The cultured enteric neurons were pre-treated with tocilizumab, R-7050 and IL-1ra for 12 $\mathrm{h}$ followed by M0, M1 supernatant for $24 \mathrm{~h}$. The relative expression levels of OT and OTR mRNA were analyzed by qRT-PCR. Noted that three receptor inhibitors reversed the role of M1 supernatant on the expression of OT. (E) The cultured enteric neurons were pre-treated with LY2109761for $12 \mathrm{~h}$ followed by M0, M2 supernatant for $24 \mathrm{~h}$. The relative expression levels of OT and OTR mRNA were analyzed by qRTPCR. LY2109761 down-regulated the expression of OT and OTR mRNA by inhibiting the expression of TGF- $\beta$. Values represent the mean \pm SEM of 6 samples and were compared by One-way ANOVA with Newman-Keuls and Dunnett for multiple comparisons. ${ }^{*} p<0.05,{ }^{* \star} p<0.01,{ }^{\star \star \star} p<0.001$. 

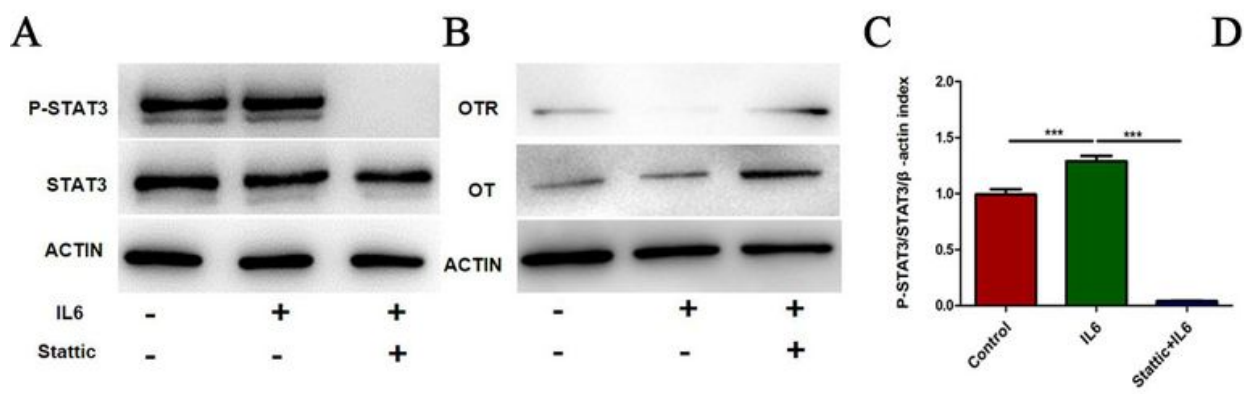

D
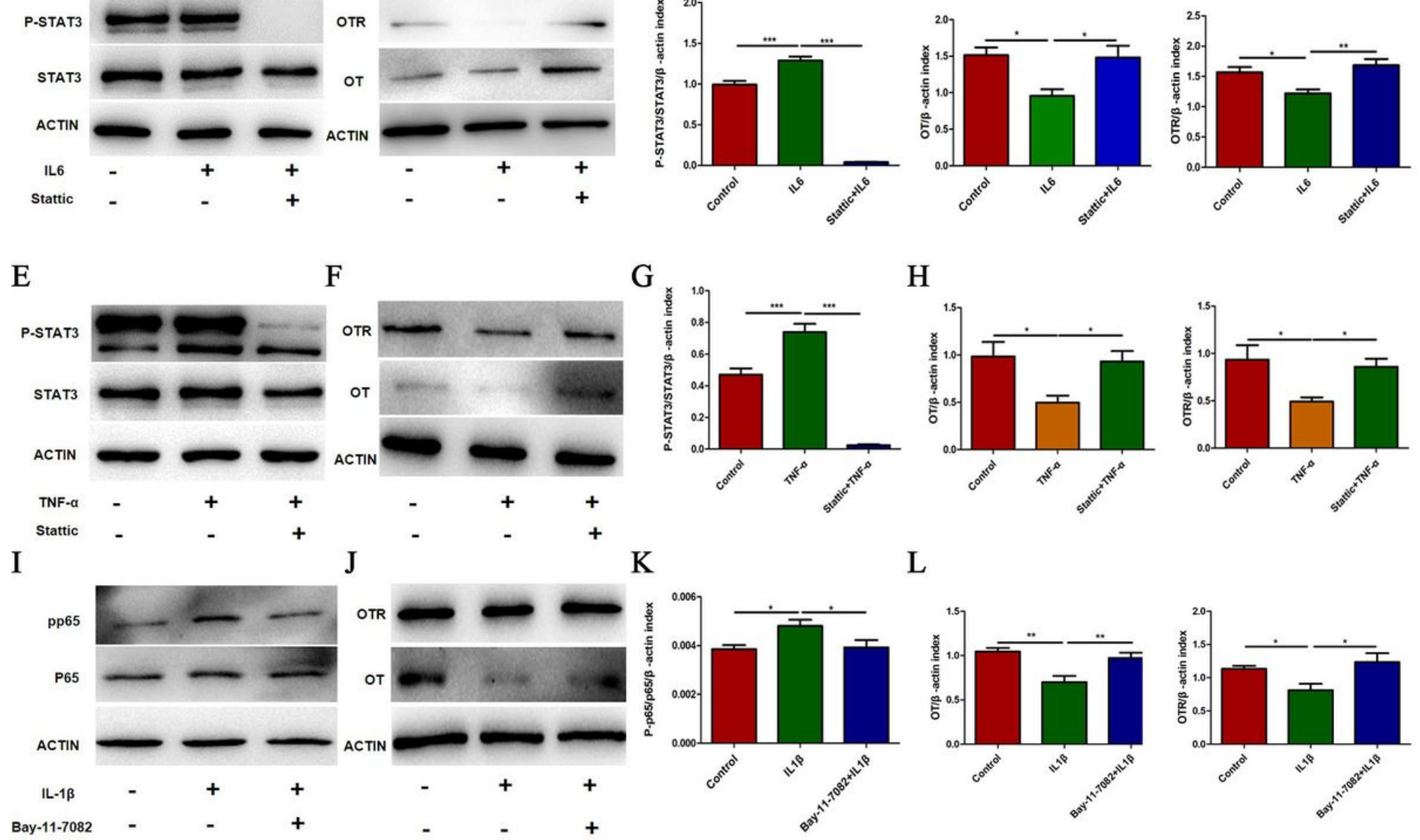

$\mathrm{H}$
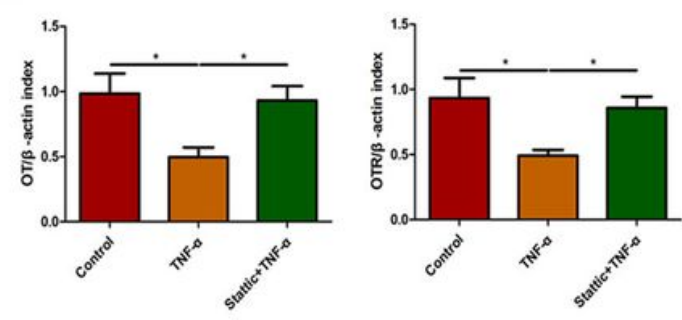

L
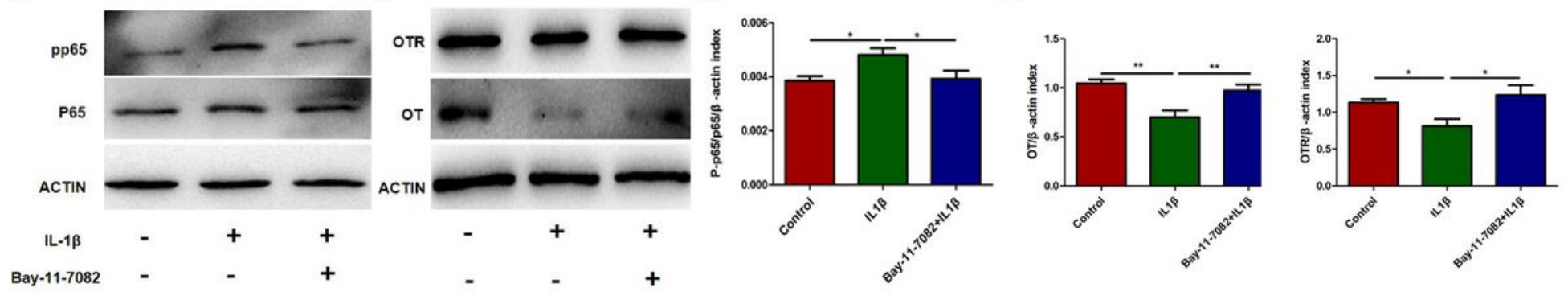

\section{Figure 4}

Inflammatory cytokines downregulated expression of OT/OTR proteins in enteric neurons via STAT3 or NF-кB pathway. (A, B, C, D) The cultured enteric neurons were pre-treated with or without Stattic (STAT3 inhibitor, $5 \mu \mathrm{M})$ for $12 \mathrm{~h}$ followed by IL6 $(10 \mathrm{ng} / \mathrm{ml})$ or TNF-a $(20 \mathrm{ng} / \mathrm{ml})$ for $24 \mathrm{~h}$. The levels of phosphorylated STAT3 (p-STAT3), total STAT3, OT, OTR were analyzed with use of western blots and $\beta$ actin was used to evaluate protein loading. IL-6 $(10 \mathrm{ng} / \mathrm{ml})$ or TNF-a $(20 \mathrm{ng} / \mathrm{ml})$ decreased expression of OT and OTR by activating STAT3 pathway. (E, F) The cultured enteric neurons were pre-treated with or without Bay11-7082 (NF-KB inhibitor, $1 \mu \mathrm{M}$ ) for $2 \mathrm{~h}$ followed by IL-1 $1 \mathrm{ng} / \mathrm{ml}$ ) for $24 \mathrm{~h}$. The levels of phosphorylated NF-KB (p-p65), total NF-KB (p65), ОT, OTR were analyzed with use of western blots and $\beta$ actin was used to evaluate protein loading. IL-1 $(1 \mathrm{ng} / \mathrm{ml})$ decreased expression of OT and OTR by activating NF-KB pathway. Values represent the mean \pm SEM of 6 samples and were compared by Oneway ANOVA with Newman-Keuls for multiple comparisons. ${ }^{\star} p<0.05,{ }^{\star} * p<0.01,{ }^{\star} * \star p<0.001$. 
A

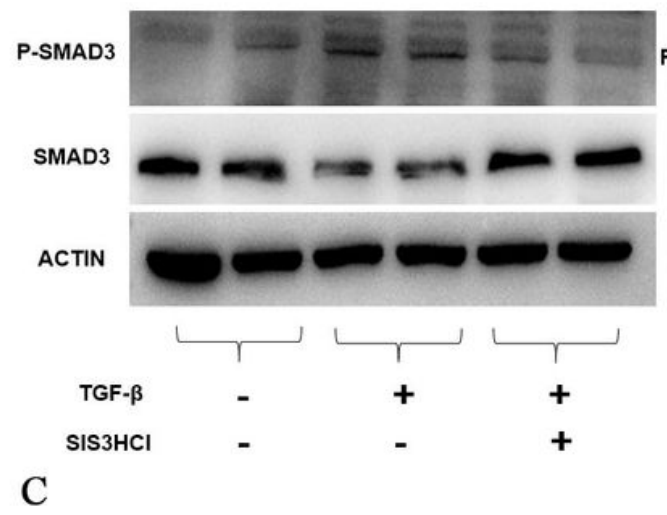

P-SMAD2

SMAD2

ACTIN

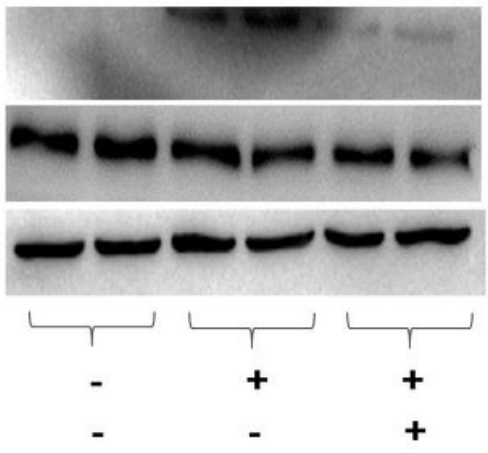

B
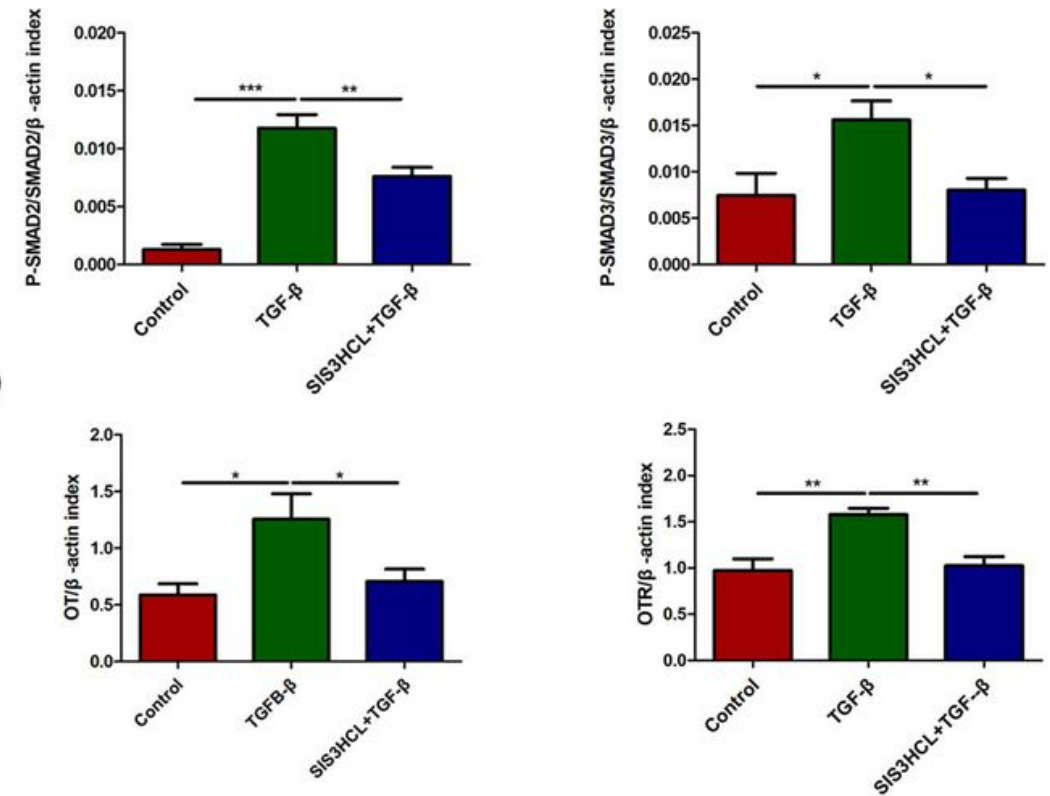

$\mathrm{D}$

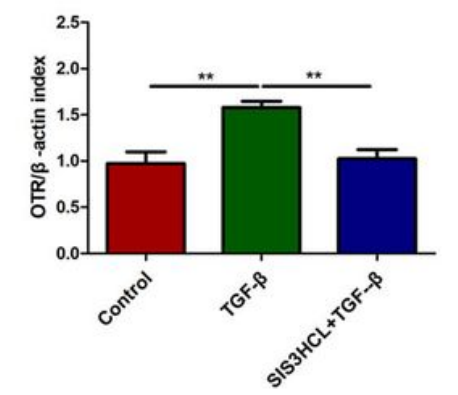

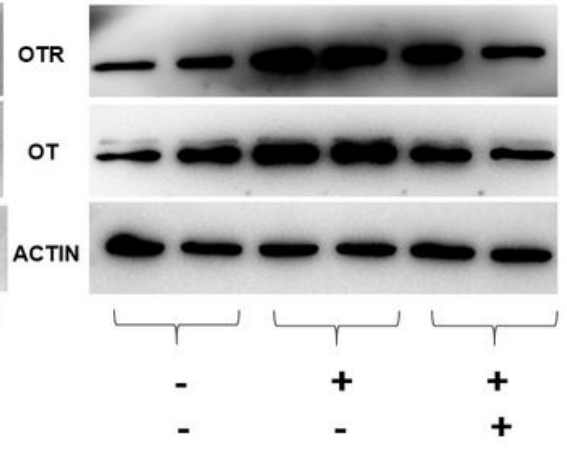

\section{Figure 5}

Smad2/3 pathway was involved in the upregulation of OT/OTR expressions following TGF- $\beta$. (A, B, C, D) The cultured enteric neurons were pre-treated with or without SIS3CHL (Smad3 inhibitor, 5 $\mathrm{M}$ ) for $12 \mathrm{~h}$ followed by TGF- $\beta$ (10 ng/ml) for $24 \mathrm{~h}$. The levels of phosphorylated Smad2 (p-Smad2), phosphorylated Smad3(p-Smad3), total Smad2, total Smad3, OT, OTR were analyzed with use of western blots and $\beta$ actin was used to evaluate protein loading. TGF- $\beta(10 \mathrm{ng} / \mathrm{ml})$ increased expression of OT and OTR by activating Smad2/3 pathway. Values represent the mean \pm SEM of 6 samples and were compared by One-way ANOVA with Newman-Keuls for multiple comparisons. ${ }^{*} p<0.05,{ }^{\star *} p<0.01,{ }^{\star \star *} p<0.001$. 
A

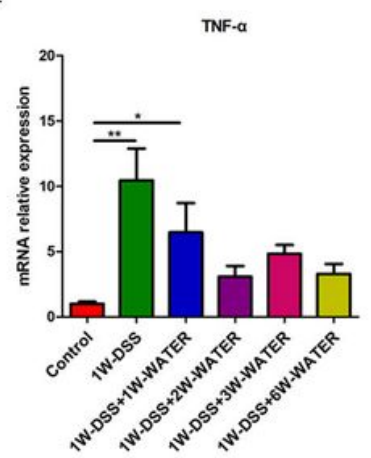

E

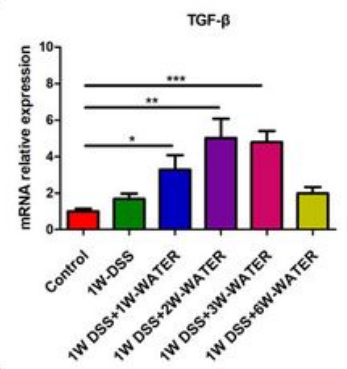

I

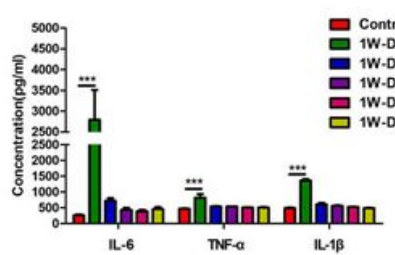

B

C

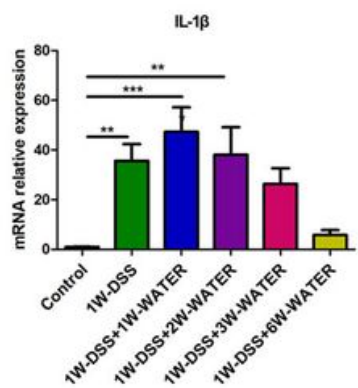

G

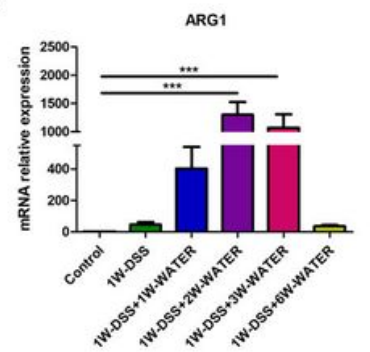

K

J
D

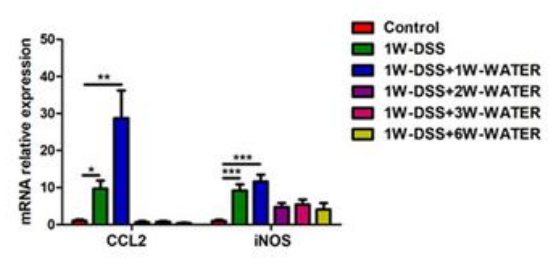

H

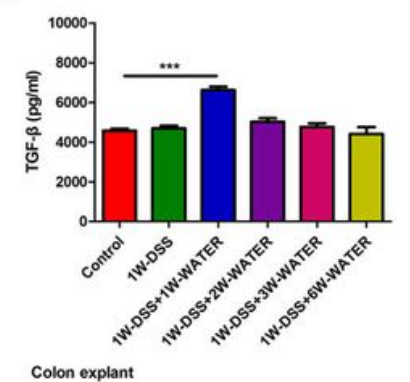

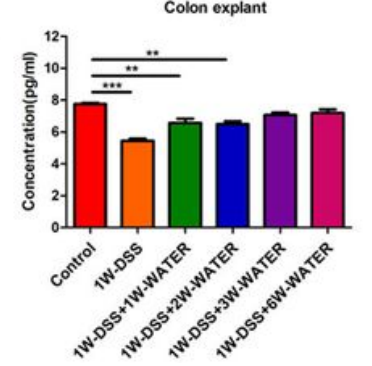

Figure 6

Correlationship between macrophages polarization and expression of OT and OTR in DSS-induced colitis. $(A, B, C, D)$ The levels of TNF- $a, I L-6, I L-1 \beta, C C L 2$, iNOS mRNA of LMMP were measured in different periods of the DSS model by qRT-PCR. In the first and second weeks, the levels of IL-1 $\beta$, IL-6, TNF-a, CCL2, iNOS mRNA were up-regulated. The above results suggested that $M 1$ macrophages are polarized. $(E, F, G)$ The levels of TGF- $\beta$, CD206, ChIL3, Arg1 mRNA of LMMP were measured in different periods of the DSS model by qRT-PCR. From the third week of the model, the levels of TGF- $\beta$, CD206, ChIL3, Arg1 mRNA were up-regulated. The results suggested that M2 macrophages are polarized. $(H, I)$ The levels of IL-6, TNF- $a$, IL-1 $\beta$ and TGF- $\beta$ proteins were measured in LMMP by ELISA. $(J)$ The levels of OT, OTR mRNA of LMMP were measured in different periods of the DSS model by qRT-PCR. The expression of OT and OTR decreased as inflammation occurs, and increased as inflammation subsides. (K) The level of OT protein in colon explant was measured in different periods of the DSS model by ELISA. Values represent the mean \pm SEM of 7 samples and were compared by One-way ANOVA with Dunnett for multiple comparisons. ${ }^{*} p<0.05,{ }^{* \star} p<0.01,{ }^{\star \star *} p<0.001$. 
A

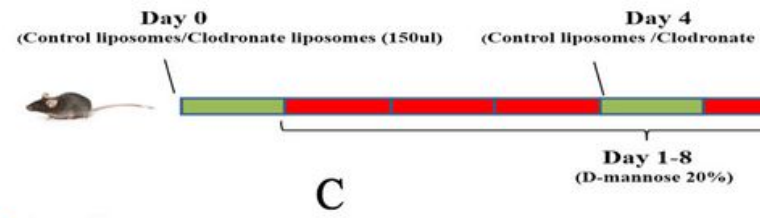

B

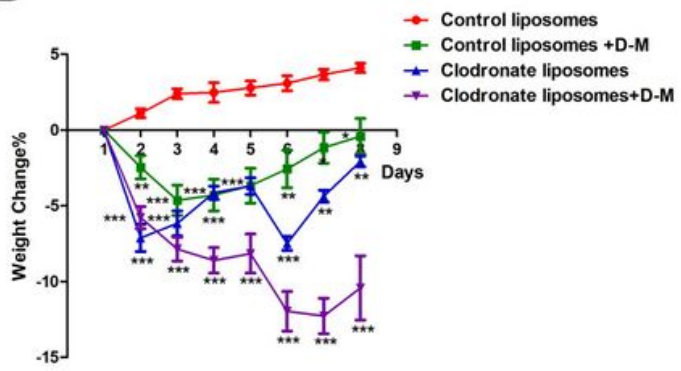

$\mathrm{E}$

F4-80

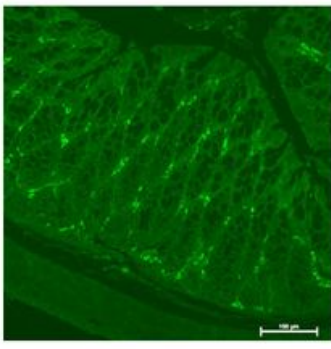

Control liposomes

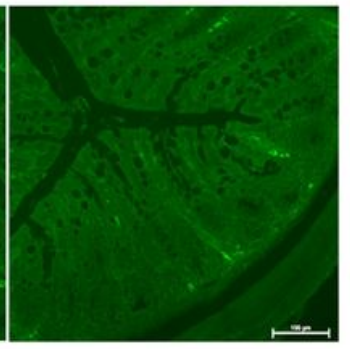

Clodronate liposomes
$\mathrm{H}$

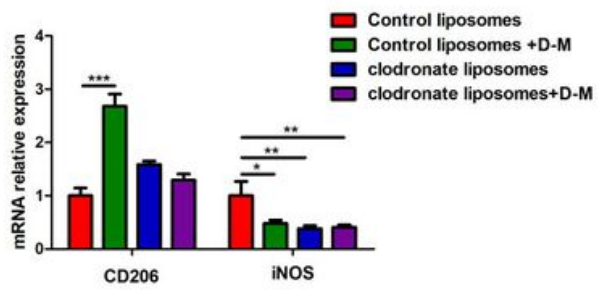

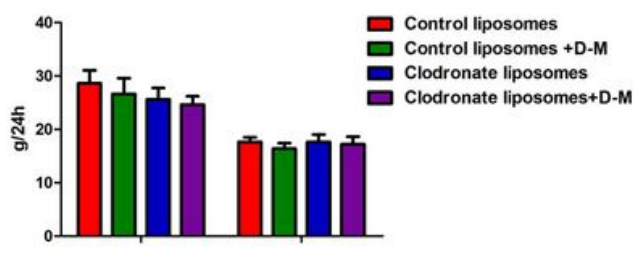

$\mathrm{F}$
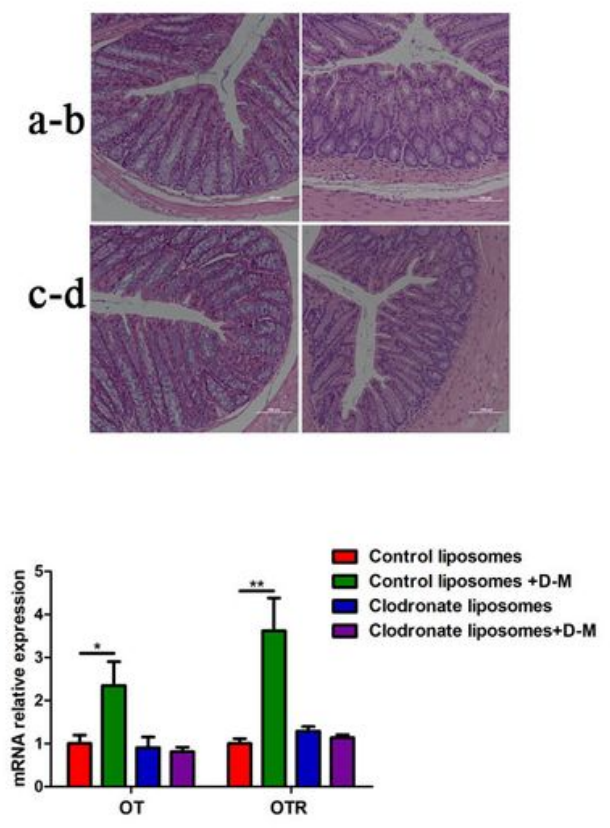

D
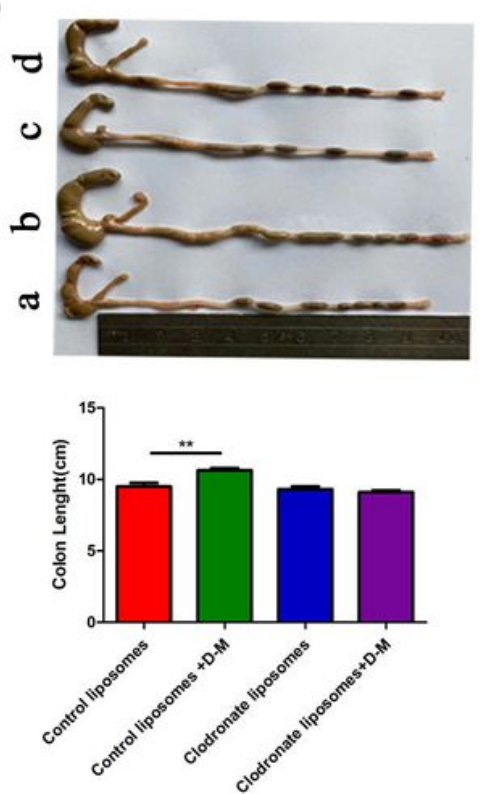

I

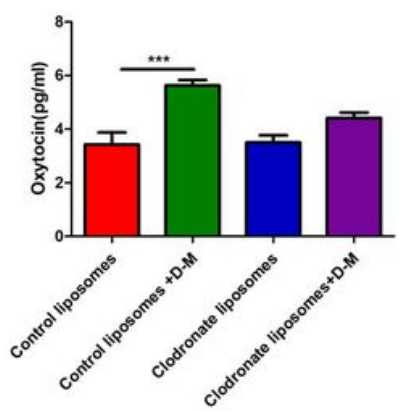

Figure 7

M2 macrophage polarization increased the expression of OT and OTR in colon. (A) Animal pattern. The day before and on the fourth day of the D-mannose model, clodronate liposomes/control liposomes (200 $\mu \mathrm{L} /$ mouse) were injected intraperitoneally, and $20 \% \mathrm{D}$-mannose was drunk daily for 7 consecutive days. (B, C) Body weight and eating habits of mice had no change during the 7 days D-mannose model. (D) The lengths of the colon from different groups were statistically compared. (E) Colonic tissue sections from the indicated groups were stained with anti-F4-80 antibody respectively to detect macrophages numbers (Scale bar: $100 \mu \mathrm{m}, \mathrm{N}=4$ ). (F) Representative H\&E staining colonic section in each group (Scale bar: $100 \mu \mathrm{m}, \mathrm{N}=4$ ). (G) The levels of CD206 and iNOS mRNA were measured after injected control liposomes and clodronate liposomes by qRT-PCR. $(\mathrm{H})$ The levels of OT, OTR mRNA of LMMP were measured after injected control liposomes and clodronate liposomes by qRT-PCR. Noted that after macrophages were depleted, the expression of OT and OTR decreased significantly. (I) The concentration of OT in plasma was measured after injected control liposomes and clodronate liposomes by ELISA. Values represent the mean \pm SEM of 6 samples and were compared by One-way ANOVA with Dunnett for multiple comparisons. ${ }^{*} \mathrm{p}<0.05,{ }^{* *} \mathrm{p}<0.01,{ }^{* * *} \mathrm{p}<0.001$. 
A

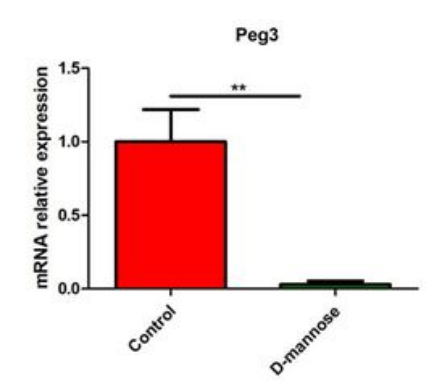

B

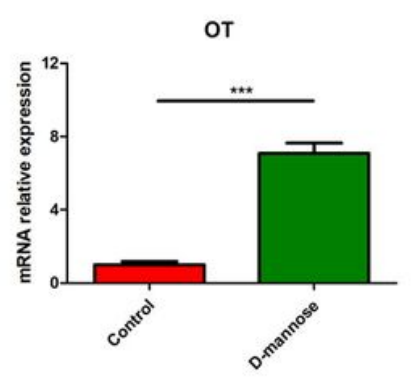

D

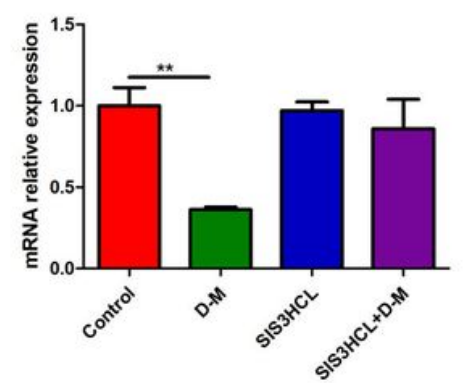

$\mathrm{E}$

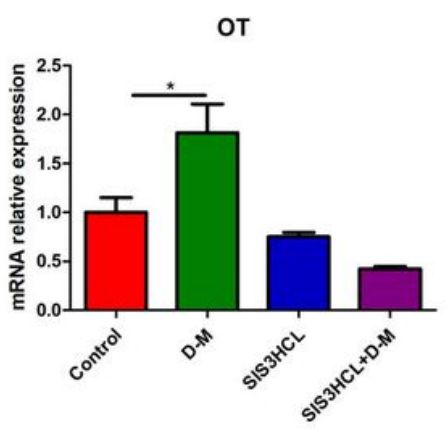

$\mathrm{C}$

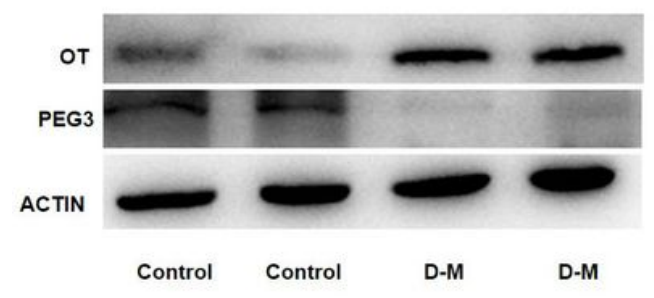

G

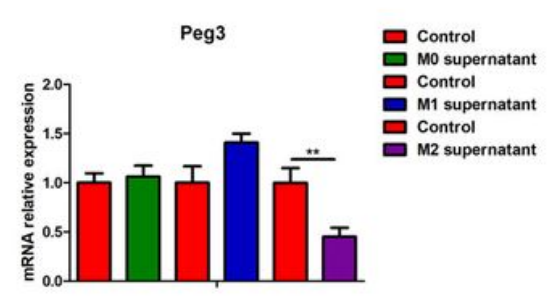

$\mathrm{H}$

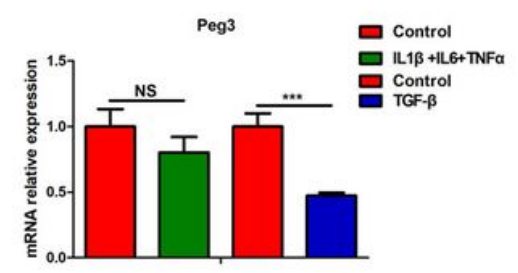

$\mathrm{F}$
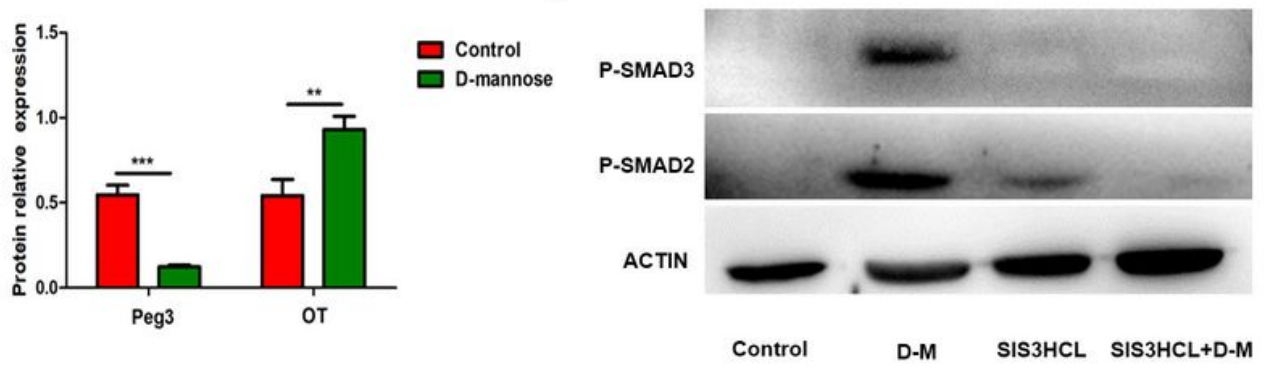

I

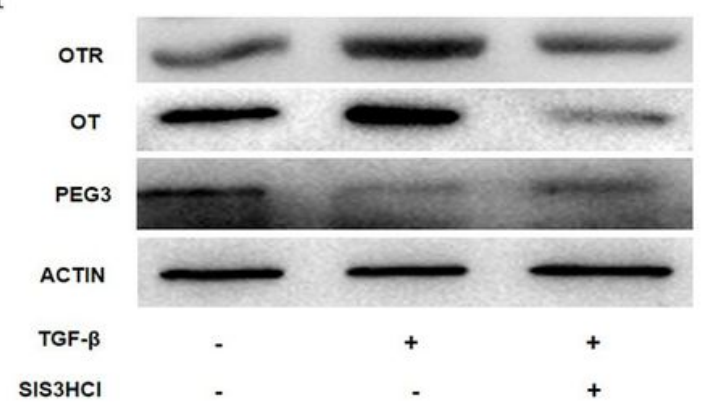

Figure 8

Peg3 was involved in the up-regulation of OT and OTR expression induced by M2 macrophages polarization. (A) The level of Peg3 mRNA of LMMP in D-mannose model was measured by qRT-PCR. The expression of Peg3 of LMMP in the D-mannose group was significantly down-regulated compared with the control group. $(B, C)$ Noted that the levels of OT mRNA and protein of LMMP in D-mannose model were upregulated with qRT-PCR and western blots analysis. (D, E) The levels of Peg3 and OT mRNA of LMMP in D-mannose model were measured after injected SIS3HCL (Smad3 inhibitor, $2.5 \mu \mathrm{g} / \mathrm{g}$ ) by qRTPCR. (F) Representative immunoblots were detected for phosphorylated Smad2/3 after injected SIS3HCL with western blots analysis and $\beta$-actin was used to evaluate protein loading. (G) The level of Peg3 mRNA in cultured enteric neurons with or with conditioned medium was measured by qRT-PCR. Noted that M2 supernatant suppressed the expression of Peg3. $(\mathrm{H})$ The level of Peg3 mRNA in cultured enteric neurons with or with IL-1 $\beta(1 \mathrm{ng} / \mathrm{ml})$, IL-6 $(10 \mathrm{ng} / \mathrm{ml})$, TNF-a $(20 \mathrm{ng} / \mathrm{ml})$ or TGF- $\beta(10 \mathrm{ng} / \mathrm{ml})$ was measured by qRTPCR. However only TGF- $\beta$ inhibited the expression of Peg3. (I) The cultured enteric neurons were pretreated with SIS3CHL (Smad3 inhibitor, $5 \mu \mathrm{M})$ for $12 \mathrm{~h}$ followed by TGF- $\beta(10 \mathrm{ng} / \mathrm{ml}$ ) for $24 \mathrm{~h}$. The levels of Peg3, OT, OTR proteins were analyzed with use of western blots and $\beta$-actin was used to evaluate 
protein loading. Values represent the mean \pm SEM of 6 samples and were compared by t-test or One-way ANOVA with Dunnett for multiple comparisons. ${ }^{*} p<0.05,{ }^{* *} p<0.01$, ${ }^{* *} p<0.001$.

M1
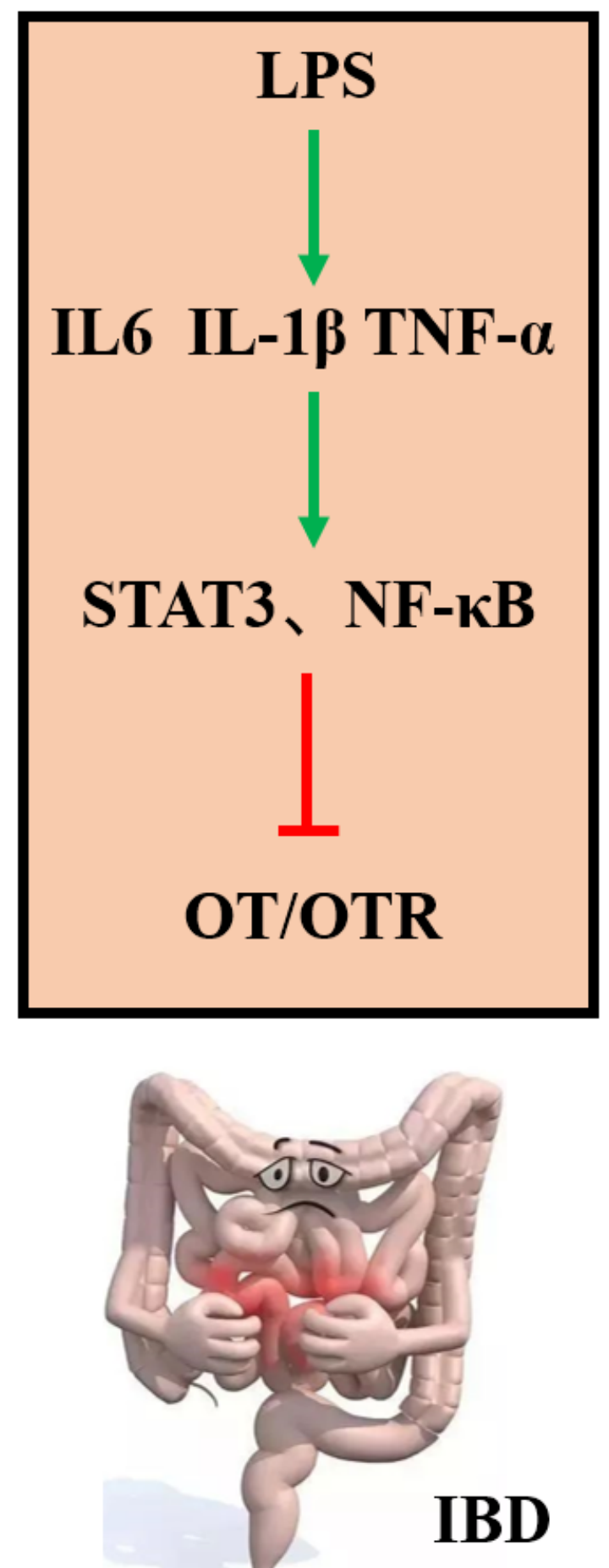

M2
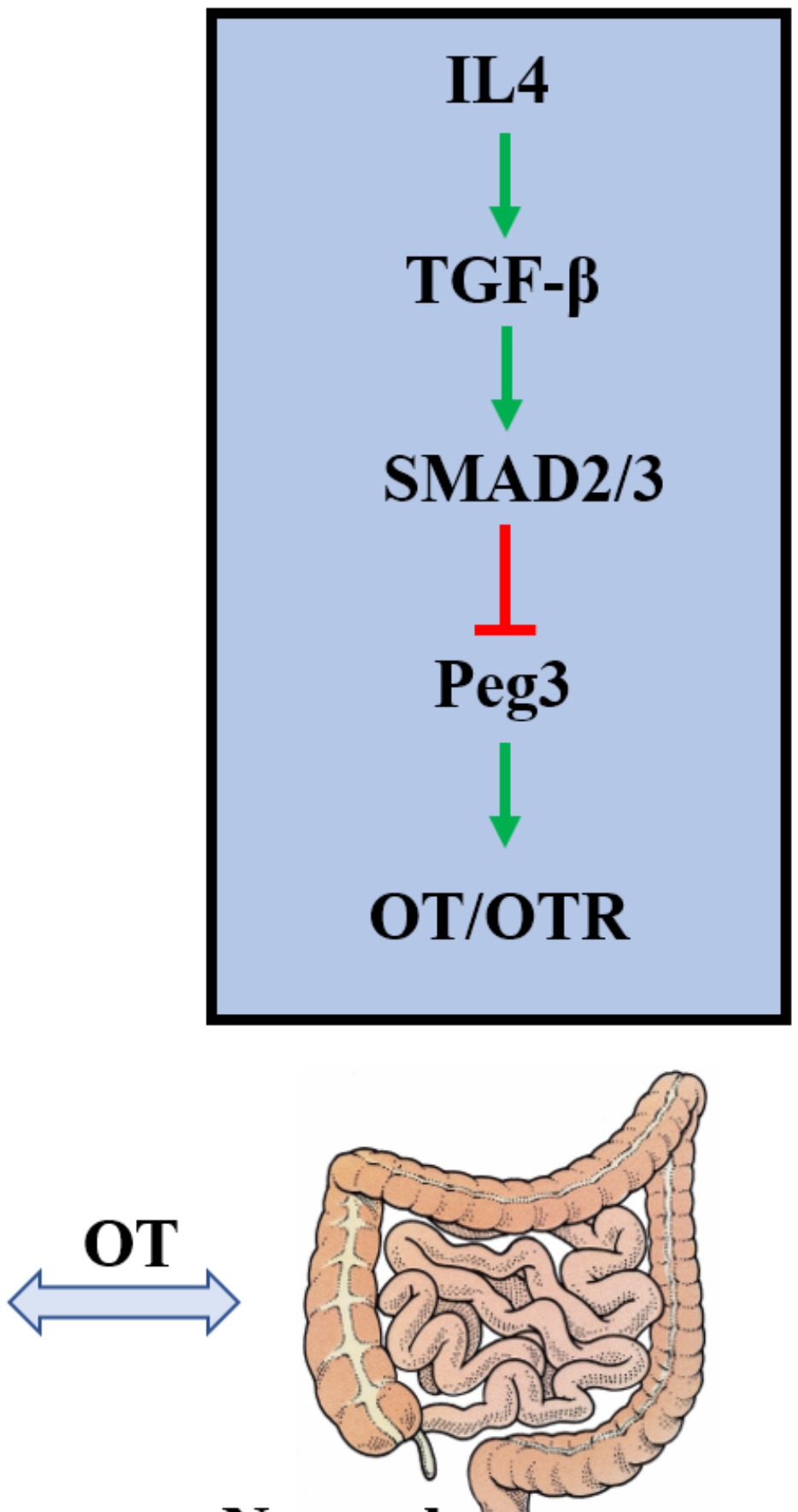

Normal

\section{Figure 9}

The mechanism of macrophages polarization modulated the OT signaling system. LPS-induced M1-like polarization to secrete pro-inflammatory factors activated STAT3 and NF-KB pathways to inhibit OT signaling system in enteric neurons. IL4-induced M2-like polarization to secrete anti-inflammatory factors 
activated Smad2/3 pathways to accelerate OT signaling system by inhibiting the expression of Peg3 in the enteric neurons. OT may be the potent chemical for the treatment of gastrointestinal disorders caused by immune dysfunction.

\section{Supplementary Files}

This is a list of supplementary files associated with this preprint. Click to download.

- Additionalfiles.docx 